\title{
Kuiper Belt Objects: Relics from the Accretion Disk of the Sun
}

\author{
Jane X. Luu \\ Lincoln Laboratory, Massachusetts Institute of Technology, Lexington, Massachusetts \\ 02420; email:luu@ll.mit.edu
}

David C. Jewitt

Institute for Astronomy, University of Hawaii, Honolulu, Hawaii 96822;

email: jewitt@ifa.hawaii.edu

Key Words Kuiper Belt, comets, solar system formation

Abstract The Kuiper Belt consists of a large number of small, solid bodies in heliocentric orbit beyond Neptune. Discovered as recently as 1992, the Kuiper Belt objects (KBOs) are thought to hold the keys to understanding the early solar system, as well as the origin of outer solar system objects, such as the short-period comets and the Pluto-Charon binary. The KBOs are probably best viewed as aged relics of the Sun's accretion disk. Dynamical structures in the Kuiper Belt provide evidence for processes operative in the earliest days of the solar system, including a phase of planetary migration and a clearing phase, in which substantial mass was lost from the disk. Dust is produced to this day by collisions between KBOs. In its youth, the Kuiper Belt may have compared to the dust rings observed now around such stars as GG Tau and HR 4796A. This review presents the basic physical parameters of the KBOs and makes connections with the disks observed around nearby stars.

\section{INTRODUCTION}

The perceived extent of the planetary region has changed greatly in the last few centuries. The planets out to Saturn (at $10 \mathrm{AU}$ ) are visible to the naked eye and were known to the ancients. Uranus (20 AU) and Neptune (30 AU) are fainter and their discoveries, in 1781 and 1846, respectively, required the aid of telescopes. Pluto, whose semimajor axis is 39 AU but whose eccentric orbit brings it to perihelion at $28 \mathrm{AU}$, was discovered photographically in 1930 following dynamical calculations purportedly accounting for deviations in the motion of Neptune (these deviations are now known to have been spurious). Very recently, observations with highly sensitive charge-coupled device (CCD) detectors have revealed a vast swarm of bodies beyond Neptune (Figure 1), now often referred to as the Kuiper Belt. Continued work has shown that Pluto is simply the largest known of these trans-Neptunian objects and that its original 
classification as a planet obscures its true significance as a member of the Kuiper Belt.

At the same time, the new discoveries have shown that the Solar System still retains a great many bodies that may be relatively unaltered products of accretion in the circumsolar disk. The Kuiper Belt itself is a remnant of the solar nebula, with its larger members primordial fossils that have escaped collisional disruption or ejection from the solar system. In addition, the Belt is most likely the common link between our solar system and other younger planetary systems. The Belt is sufficiently populated that its members collide with each other, and these collisions have generated dust since the Belt's earliest days. This collisionally produced dust should form a large, albeit distended, disk surrounding the planetary region, thus providing our solar system with its own circumstellar disk - much like a local analog of the dusty disks around some young and main-sequence stars. Indeed, future technology might one day detect the extrasolar analogs of Kuiper Belt bodies in these external systems.

A number of individuals speculated in print in the early twentieth century about the possible existence of objects beyond the planetary region. These included the Irish aristocrat Kenneth Edgeworth (Edgeworth 1943, 1949; see also MacFarland 1996). Musing about the origin of short-period comets, Edgeworth suggested that they might originate beyond Pluto-a hypothesis that is now widely accepted. Later, the Dutch-American astronomer Gerard Kuiper (1951) discussed a ring of small bodies beyond Pluto, the latter thought by him to be a massive planet (its mass is, in fact, $0.002 \mathrm{M}_{\oplus}$ where $\mathrm{M}_{\oplus}=6 \times 10^{24} \mathrm{~kg}$ is the mass of the Earth). He postulated that small, comet-sized bodies might have formed in the transNeptunian region, subsequently to be scattered to the Oort cloud by Pluto. Comets in the Oort cloud can then return to the planetary region through perturbations of passing stars (Oort 1950). In this way, Kuiper used the trans-Neptunian space to supply comets to the much more distant Oort Cloud. For reasons that remain the subject of widespread speculation, Kuiper did not refer to the prior investigations of Edgeworth. Finally, neither Edgeworth nor Kuiper cited the even earlier writing of Leonard (1930), who correctly speculated that Pluto would prove to be the first of many objects to be discovered beyond Neptune. Was the Kuiper Belt predicted? In the modern sense in which a physically based theory is required to make observationally testable predictions, the answer is clearly "no." However, several people correctly guessed that the region beyond Pluto might not be empty.

The Kuiper Belt is sometimes also referred to as the Edgeworth-Kuiper Belt in an effort to recognize the contribution of Edgeworth (perhaps the LeonardEdgeworth-Kuiper Belt is not far behind). However, in this review, for the sake of brevity, we will use the name Kuiper Belt and refer to members of the Belt as Kuiper Belt objects or KBOs for short. Other general reviews of the KBOs may be found in Jewitt (1999), Jewitt \& Luu (2000), and Schulz (2001). Reviews of specific aspects include (dynamics) Malhotra et al. (2000) and (collisions) Farinella et al. (2000). A technical Kuiper Belt book has been published (Fitzsimmons et al. 2000), as has a popular one (Davies 2001). 


\section{STRUCTURE OF THE KUIPER BELT}

Figure 2 depicts the KBO orbits as seen from the North ecliptic pole. At the time of writing (August 2001), about $400 \mathrm{KBOs}$ have been reported, the results of several telescopic surveys carried out all over the world. The interested reader is referred to the Web sites below for $\mathrm{KBO}$ orbits and additional information:

http://cfa-www.harvard.edu/cfa/ps/lists/TNOs.html

$\mathrm{ftp} / / / \mathrm{ftp}$. lowell.edu/pub/elgb/astorb.html

http://www.ifa.hawaii.edu/faculty/jewitt/kb.html

Neptune (semimajor axis $a=30 \mathrm{AU}$ ) defines the inner edge of the Kuiper Belt. Almost all the known KBOs were found beyond Neptune, most of them with semimajor axes between 30 and $50 \mathrm{AU}$, although a few, the Scattered KBOs, have semimajor axes of hundreds of $\mathrm{AU}$ (see below). It is also clear that the Kuiper Belt possesses a complex structure: The KBOs are not spread out uniformly in the Belt, but are clustered into subgroups, attesting to the fact that the Belt has been extensively sculpted by the gravitational influence of the giant planets (particularly Neptune). From the orbits of known KBOs, three distinct dynamical subgroups have emerged: (a) the Classical KBOs, $(b)$ the Resonant KBOs, and (c) the Scattered KBOs. The details that distinguish the KBO dynamical classes can be seen in Figures 3 and 4, and are discussed below.

\section{Classical KBOs and the Inclination Distribution}

The Classical KBOs make up the majority ( $\sim$ two-thirds) of known KBOs, and are characterized by semimajor axes $42<a<48 \mathrm{AU}$, relatively small eccentricity $e(e \sim 0.1$, Figure 3$)$, perihelia $q>35 \mathrm{AU}$, and the fact that they lie outside resonances. Numerical simulations show that objects with such large semimajor axes and perihelia are relatively immune to Neptune's gravitational influence on Gyr timescales (Holman \& Wisdom 1993, Duncan et al. 1995, Morbidelli et al. 1995). It was initially suspected that the Classical KBOs formed in situ and have largely kept their primordial orbits, but the unexpectedly broad inclination $(i)$ and eccentricity (e) distributions (see Figures 3 and 4 ) show that the orbits have been excited since formation.

The inclination distribution of the KBOs is a measure of the velocity dispersion among these objects and gives lie to the excited state of the Kuiper Belt. Measurement of the inclination distribution is made difficult by observational bias: Most surveys to date have been conducted near the ecliptic, but the fraction of each orbit a KBO spends near the ecliptic scales roughly as the inverse of its inclination, so that high-inclination KBOs are discriminated against by these surveys. Furthermore, efforts to map the thickness of the Kuiper Belt are hindered by the Belt's relatively low off-ecliptic surface density. Detection of KBOs with $i \sim 30^{\circ}$ in early ecliptic surveys already showed that the inclination distribution must be broad 


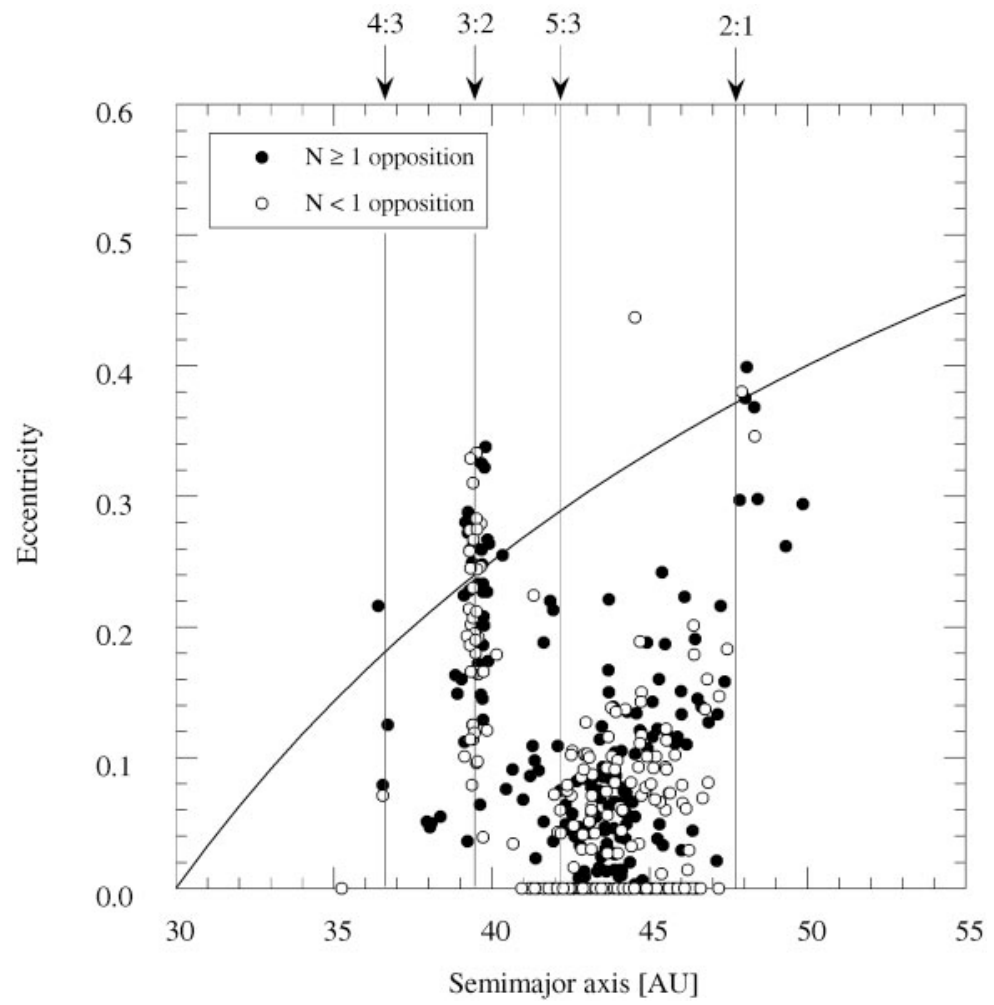

Figure 3 Semimajor axis $a$ vs. eccentricity $e$ for Classical and Resonant KBOs. Filled circles denote multi-opposition orbits, open circles denote orbits computed from astrometry taken within a single opposition. The curve marks perihelion $q=30$ AU; objects above the line are Neptune-crossers. Vertical lines mark the locations of mean-motion resonances with Neptune. Orbits with $e=0$ are assumed, not accurately measured.

(Jewitt et al. 1996), with a Half Width at Half Max (HWHM) $\Delta i \geq 20^{\circ}$. Subsequently, a very large-format 12k-CCD camera (12 edge-abutted $4096 \times 2048$ pixel CCDs, Cuillandre et al. 2000) on the Canada-France-Hawaii Telescope (CFHT) made possible targeted observations at ecliptic latitudes $0^{\circ}, 10^{\circ}$, and $20^{\circ}$, yielding $\Delta i \sim 20^{\circ}{ }_{-4}^{\circ}(1 \sigma)$, (Trujillo et al. 2001a, Brown 2001). This thick disk is very different from the one in which the KBOs are believed to have accreted (Kenyon \& Luu 1999a). The mean velocity dispersion is thus $\Delta V \approx 1.2 \mathrm{~km} \mathrm{~s}^{-1}$, which is large compared to the escape velocities of all but the largest KBOs.

A number of hypotheses have been offered to explain the relatively high $e$ 's and $i$ 's of the Classical KBOs, such as scattering by Earth-sized bodies in the early Kuiper Belt (Morbidelli \& Valsecchi 1997), and partial trapping by sweeping of the 2:1 resonance (Hahn \& Malhotra 1999). Alternatively, orbits of KBOs beyond 


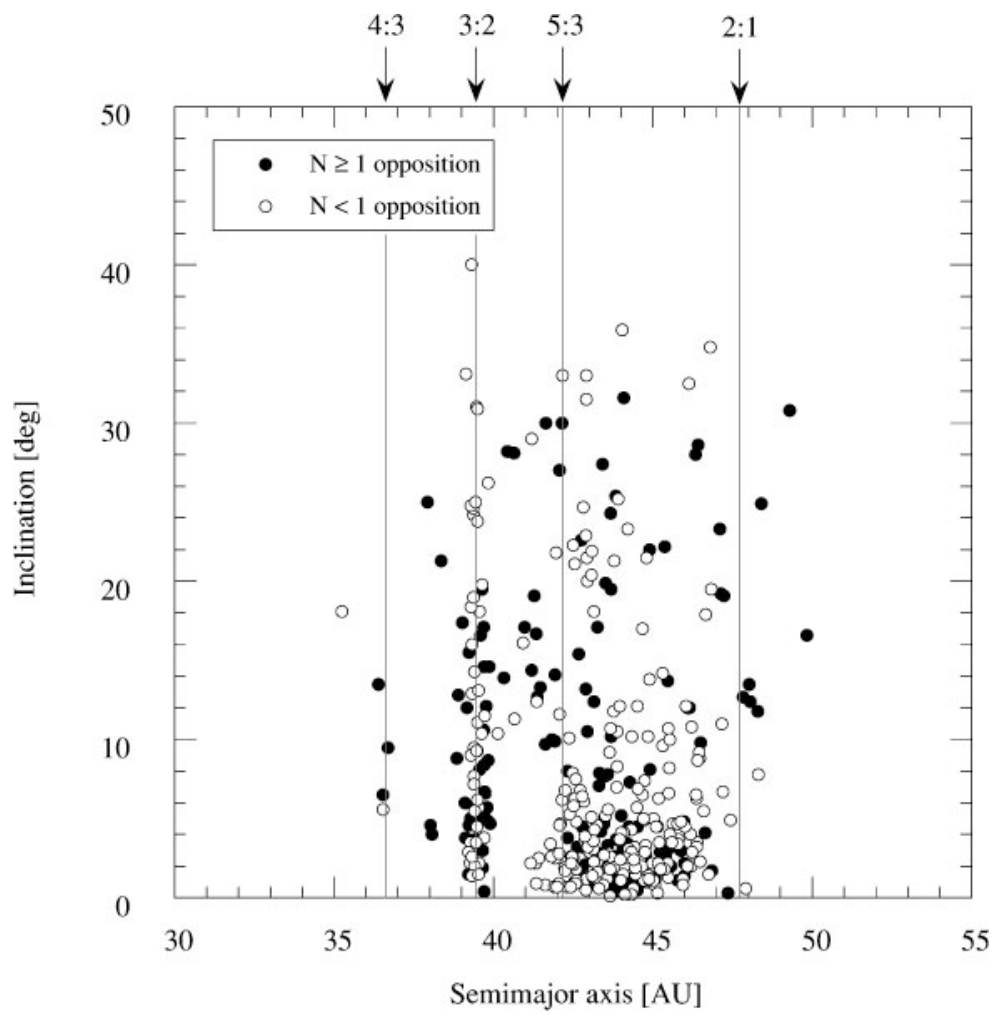

Figure 4 Inclination $i$ vs. semimajor axis $a$ for Classical and Resonant KBOs. Filled circles denote multi-opposition orbits, open circles denote orbits computed from astrometry taken within a single opposition. Vertical lines mark the locations of meanmotion resonances with Neptune.

42 AU may have been excited by stellar encounters with periapse on the order of 150-200 AU (Ida et al. 2000b), or by sweeping secular resonances (Nagasawa \& Ida 2000). The former hypothesis also predicts that $e$ and $i$ rapidly increase beyond $50 \mathrm{AU}$ (Figure 5), inhibiting planetesimal growth and thus producing a steep drop in the KBO number density. This seems consistent with the observed boundary of Classical KBOs at 50 AU (see "Radial Extent of the Classical Kuiper Belt" below), but this hypothesis, like the others, needs further testing. Luckily, all these models predict different distributions of $e$ and $i$, so observations targeted at the region beyond $50 \mathrm{AU}$ should allow meaningful discrimination between them.

\section{Resonant KBOs}

The Resonant KBOs occupy mean motion resonances with Neptune, i.e., their orbital periods and that of Neptune form a ratio of integers. Due to the resonance dynamics, the Resonant KBOs generally have larger $e$ 's and $i$ 's than the average 


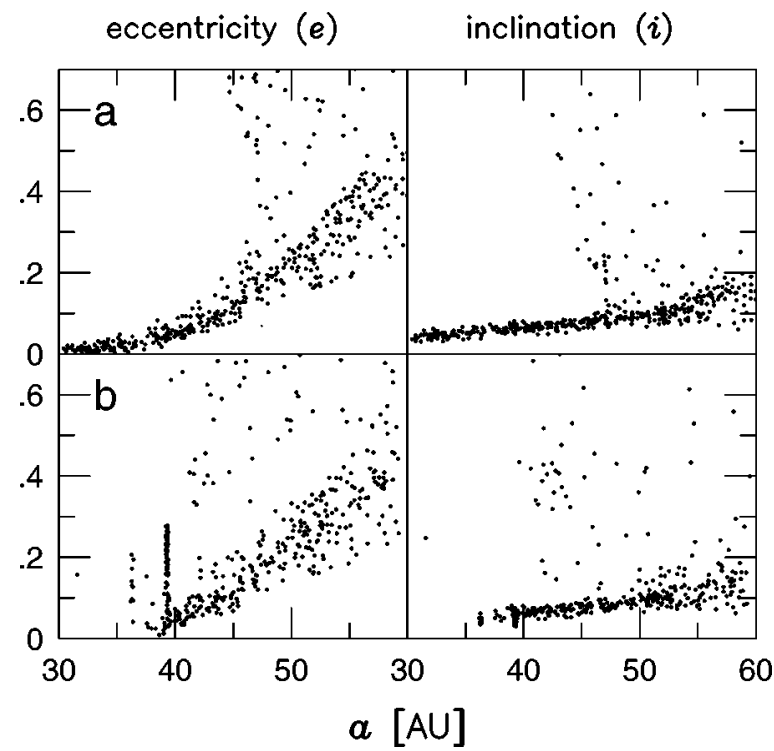

Figure 5 Predicted orbital distribution of KBOs: $(a)$ distribution after stellar encounter (before sweeping resonances), $(b)$ distribution after including sweeping resonances. Figure from Ida et al. (2000b).

Classical KBO (see Table 1). The most densely populated resonance is the 3:2, located at semimajor axis 39.4 AU (Figures 3 and 4). About $100 \mathrm{KBOs}$ are known to occupy this resonance, as does Pluto. The dynamical similarities between Pluto and the other (smaller) 3:2 objects have led to the classification of these bodies as Plutinos. Some Plutinos, like Pluto itself, have perihelia $q \leq 30$ AU, i.e., their orbits cross that of Neptune. However, like Pluto, they are protected from destabilizing close encounters with this planet by their location in the resonance (Malhotra 1995), even if their immediate environs are highly unstable (Holman \& Wisdom 1993, Levison \& Duncan 1993, Duncan et al. 1995). But not all Plutino orbits are stable over the age of the solar system. Depending on their eccentricities, some can be driven out of the 3:2 resonance by Pluto and into close encounters with

TABLE 1 Mean orbital elements of KBOs

\begin{tabular}{lccc}
\hline Dynamical group & $\boldsymbol{a}[\mathrm{AU}]$ & $\overline{\boldsymbol{e}}$ & $\overline{\boldsymbol{i}}$ [deg] \\
\hline Classical & $>42$ & 0.09 & 7.11 \\
4:3 resonance & 36.4 & 0.22 & 7.81 \\
3:2 resonance & 39.4 & 0.36 & 12.87 \\
2:1 resonance & 47.8 & 0.14 & 9.83 \\
Scattered & $>30$ & 0.49 & 14.08 \\
\hline
\end{tabular}


Neptune (Yu \& Tremaine 1999). The latter may represent one mechanism by which the Kuiper Belt supplies the short-period comets to the inner solar system.

The apparent fraction of Plutinos in the KBO sample is about $25 \%$. However, this fraction is over-estimated, the result of a discovery bias in favor of Plutinos: With smaller orbits than most other KBOs, Plutinos are, on average, brighter and over-represented in flux-limited surveys. The true abundance of Plutinos is perhaps closer to $10 \%$ (Jewitt et al. 1998, Trujillo et al. 2001a). Extrapolating to the whole sky, we estimate that there are roughly 1500 Plutinos larger than $100 \mathrm{~km}$ (see Trujillo et al. 2001a). Other mean motion resonances also appear to be populated, notably the 2:1 at 47.8 AU and the 4:3 at 36.4 AU, although small-number statistics as yet prevent their absolute populations from being determined.

How might the mean motion resonances have become populated? According to Malhotra $(1993,1995)$, the answer lies in the radial migration of the giant planets. It has long been suspected that, during the final stages of planet formation, the giant planets migrated radially due to their exchange of angular momentum with the planetesimals that they scattered (Fernandez \& Ip 1984). In this scenario, exchange of angular momentum among the gas giant planets during the diskclearing phase led to a net outward migration of Saturn, Uranus, and Neptune, at the expense of a slight inward migration of massive Jupiter (Fernandez \& Ip 1984). As Neptune moved outwards, its mean motion resonances swept ahead of it through the planetesimal disk and could have captured Pluto, along with the Plutinos, into the 3:2 resonance. The other mean motion resonances would also have captured objects from the planetesimal disk, although usually with a lower efficiency (Malhotra 1995).

The magnitude of the radial migration of Neptune can be estimated from the eccentricity of the trapped objects, via

$$
e_{f}^{2}=e_{i}^{2}+\left(\frac{1}{j+1}\right) \ln \left(\frac{a_{N, f}}{a_{N, i}}\right),
$$

where $e_{i}, e_{f}$ are the initial and final eccentricities before and after trapping in the resonance, $j$ is an integer ( $j=2$ for the $3: 2$ resonance), and $a_{N, i}$ and $a_{N, f}$ are the initial and final semimajor axes of Neptune (Malhotra 1995). Assuming $e_{i}=$ $0, a_{N, f}=30 \mathrm{AU}$ and noting that the most eccentric Plutinos have $e=0.33$ (Figure 3), we solve Equation 1 to find $a_{N, i}=21.6 \mathrm{AU}$, corresponding a total outward migration $\Delta a \approx 8$ AU.

In principle, the relative populations of different resonances can be predicted directly by the capture theory. Malhotra (1995) predicted that the populations of the $3: 2$ and 2:1 resonances should be of the same order. (We regard this prediction as noteworthy because this one was made before the key observations were in hand to test it).

However, the capture model in its initial form is highly simplistic-dynamical effects of collisions are ignored and the migration of Neptune is taken to be smooth and continuous - and depends on several unknown parameters such as the time-dependent mass of growing Neptune and the form of its migration rate (Malhotra 1995, Hahn \& Malhotra 1999). Pointing out that the resonant capture 
theory overestimated the $2: 1$ resonance population, Ida et al. (2000a) suggested that capture into the 2:1 resonance required much longer Neptune migration timescales, and explored mechanisms that might result in such long timescales. They proposed two mechanisms, involving $(a)$ the tidal effects of the solar nebula on Neptune's orbit, and (b) Neptune's close scatterings of planetesimals. Friedland (2001) proposed yet another mechanism that would inhibit capture into the 2:1 resonance: the effect of the Sun's rotation around the center of mass. The relative resonance populations determined by carefully debiased surveys might provide a method to measure the migration timescale of Neptune, and perhaps also estimate the total mass of planetesimals scattered, but the results may not be as clear-cut as was initially hoped.

\section{Scattered KBOs}

The third dynamical class in the Kuiper Belt, the Scattered KBOs (SKBOs), was established in October 1996 with the discovery of 1996 TL $_{66}$ (Luu et al. 1997). SKBOs are distinguished by their large, highly eccentric and highly inclined orbits (Figure 6). Evidently, SKBOs represent a separate type of trans-Neptunian

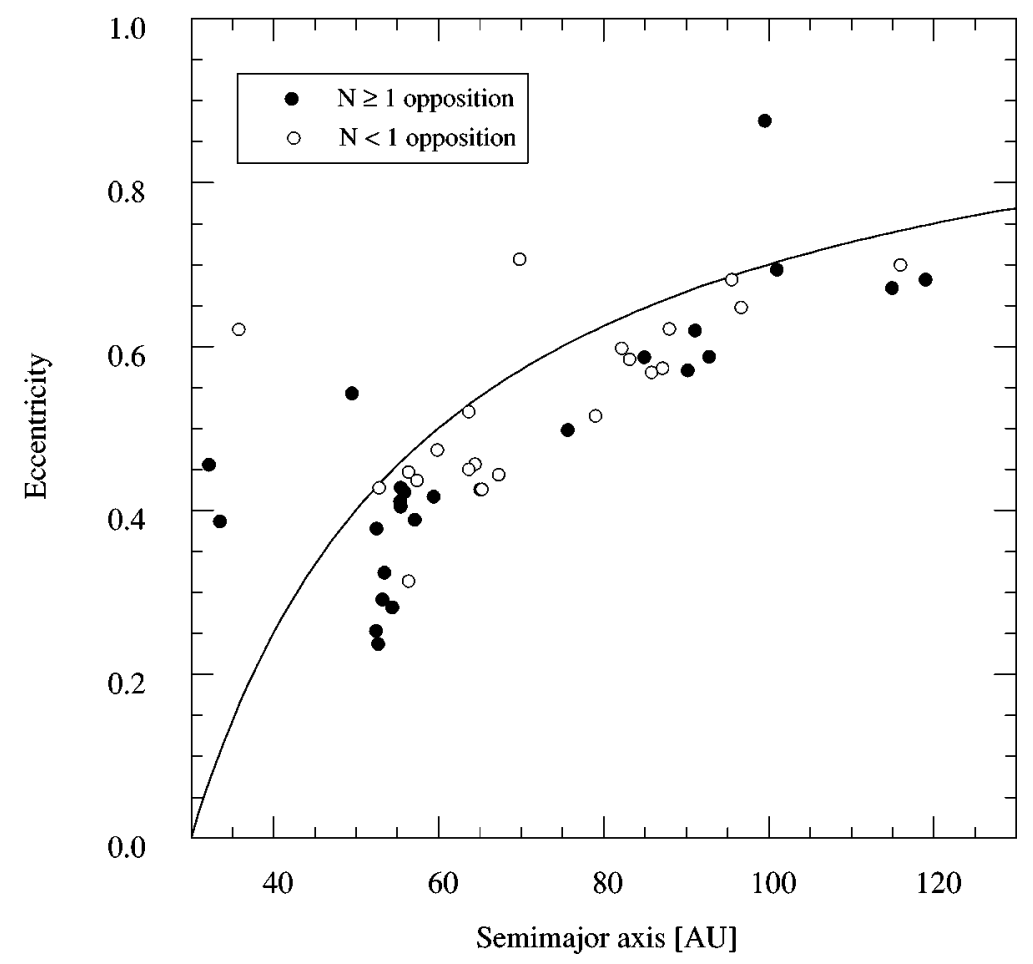

Figure $6 a$ vs. $e$ for Scattered KBOs. Same as Figure 2, but on a larger scale to show the Scattered KBOS. 
dynamics, and provide the first direct evidence that the trans-Neptunian population extends much further than the $\sim 50 \mathrm{AU}$ region sampled by previous surveys. A crude estimate of the SKBO population scattered by Neptune (with diameter $D \geq 100 \mathrm{~km}$ ) is $3.1_{-1.3}^{+1.9} \times 10^{4}$ (Trujillo et al. 2000), which is comparable to the number of the Classical KBOs. The population of SKBOs with perihelion too large to permit Neptune scattering could potentially be much larger.

The SKBOs are hypothesized to be the population of scattered planetesimals discussed earlier by Ip (1989), Torbett (1989), and Ip \& Fernandez (1991). In this scenario, during the late stages of planet formation, Uranus and Neptune scattered many of the nearby planetesimals to large distances, subsequently to form the Oort cloud. Planetesimals scattered to more modest distances formed the Scattered Kuiper Belt and remained weakly interacting with Neptune (Duncan \& Levison 1997). The recent discovery of $2000 \mathrm{CR}_{105}$, a SKBO with perihelion $(q \sim 44)$ outside the domain controlled by Neptune, raises the possibility that some SKBO orbits are not the result of direct gravitational scattering by this giant planet (B. Gladman et al., unpublished manuscript). A much larger SKBO sample is needed before firm conclusions can be drawn about their origin.

All the dynamical groups in the Kuiper Belt suffer from observational biases, but to different degrees. For example, the Plutinos are favored over the 2:1 resonant objects due to their smaller heliocentric distances, and the SKBOs are discriminated against most of all because of their very large orbits. The current best estimates of the ratios of the (bias-corrected) populations of various dynamical groups in the Kuiper Belt are as follows (Trujillo et al. 2001a):

Classical : Scattered : Plutino: $2: 1$ Resonant $=1.0: 0.8: 0.4: 0.07$

\section{POPULATION CHARACTERISTICS}

\section{Surface Density}

Published ground-based surveys of KBOs include Tombaugh (1961), Luu \& Jewitt (1988, 1998), Kowal (1989), Levison \& Duncan (1990), Irwin et al. (1995), Jewitt et al. (1996, 1998), Gladman et al. (1998), Trujillo \& Jewitt (1998), Chiang \& Brown (1999), Sheppard et al. (2000), Larsen et al. (2001), Gladman et al. (2001), and Trujillo et al. (2001a). The only survey carried out from space, using the Hubble Space Telescope at red magnitude 28.1 (Cochran et al. 1995) yielded controversial results (Brown et al. 1997, Cochran et al. 1998) and will be left out of this review.

The number most directly measured by all surveys is the cumulative surface density of KBOs, which is the number of KBOs per square degree brighter than a given apparent magnitude (Figure 7). Poisson statistics are usually assumed, and are roughly equal to Gaussian statistics for all data points representing more than a few detections. From these measurements one can calculate the cumulative 


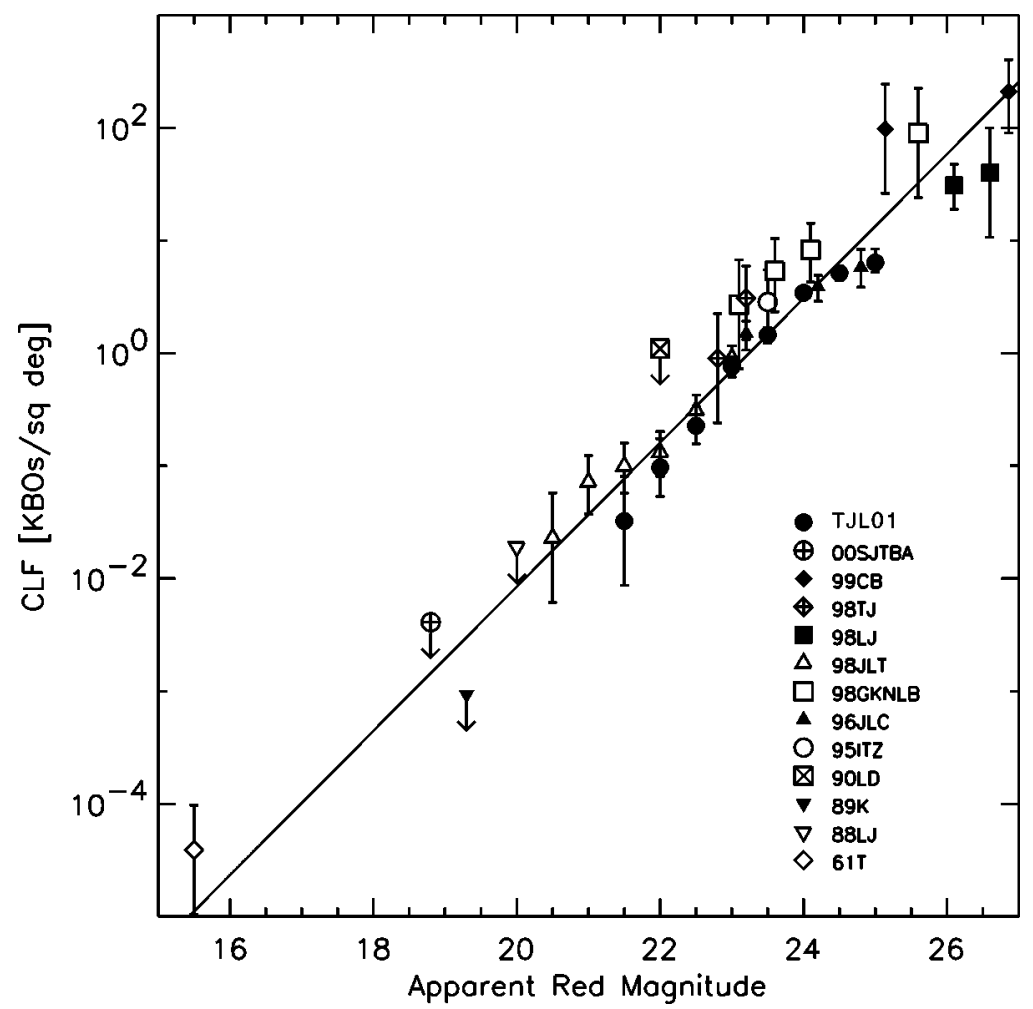

Figure 7 The cumulative surface density of KBOs. The data show the number of KBOs per square degree brighter than a given apparent red magnitude, as measured by various surveys. The legends are as follows: TJL01 = Trujillo et al. (2001a), 00SJTBA = Sheppard et al. (2000), 99CB = Chiang \& Brown (1999), 98TJ = Trujillo \& Jewitt (1998), 98LJ = Luu \& Jewitt (1998), 98JLT = Jewitt et al. (1998), 98GKNLB = Gladman et al. (1998), 96JLC = Jewitt et al. (1996), 95ITZ = Irwin et al. (1995), 90LD = Levison \& Duncan (1990), 89K = Kowal (1989), 88LJ = Luu \& Jewitt (1988), T61 = Tombaugh (1961). Figure adapted from Trujillo et al. (2001a).

luminosity function (CLF) of the Kuiper Belt by fitting the surface density $\Sigma$ with the power law

$$
\log \left[\Sigma\left(m_{R}\right)\right]=\alpha\left(m_{R}-m_{0}\right)
$$

where $m_{R}$ is the red apparent magnitude, $m_{0}$ is the (red) magnitude at which the surface density $\Sigma=1 \mathrm{deg}^{-2}$, and $10^{\alpha}$ gives the slope of the CLF. However, since the CLF at the faint end is highly correlated with that at the bright end, fitting the CLF would count the bright bodies more than once and thus underestimate the number of faint bodies. Arguably a more meaningful fit can be obtained by 
fitting not the CLF, but the differential luminosity function, which represents the number of bodies in each magnitude bin. Fitting data from our latest surveys, which span the magnitude range $m_{R}=21-26$ (Trujillo et al. 2001a), yields $\alpha=0.64_{-0.10}^{+0.11}$ and $m_{0}=23.23_{-0.20}^{+0.15}$ In other words, there is $1 \mathrm{KBO}$ per square degree at red magnitude $m_{R} \approx 23.2$, increasing by $\sim 4$ per magnitude. This result is consistent with other works (see Table 2). The luminosity function at $m_{R}<20$ and $m_{R}>26$ may be different, and remains to be assessed.

The luminosity function at $m_{R}<20$ is ill constrained due to the low surface density of bright KBOs. In spite of their scarcity, recent years have seen surveys targeted at large KBOs, such as Sheppard et al. (2000; detection limit $m_{R}=18.8$ ), Larsen et al. (2001; detection limit $m_{R} \sim 21.5$ ), Trujillo et al. (2001b; detection limit $m_{R}=21.1$ ), and the QUEST survey (Ferrin et al. 2001; detection limit $m_{R}=$ 19.6). These surveys are adding valuable data to the bright end of the KBO size distribution (Figure 7), and rule out the previously reported limit of $1 \mathrm{KBO}$ brighter than $m_{R} \sim 19.5$ per thousand square degrees of Kowal (1989).

\section{Size Distribution}

The KBOs are usually assumed to obey a differential power law size distribution of the form $n(r) d r=\Gamma r^{-q} d r$, where $n(r) d r$ is the number of objects in the radius range $r$ to $r+d r$, and $\Gamma$ and $q$ are constants. The best-fit value of $q$ (Table 2) can be determined by means of a maximum likelihood simulation of the survey, which corrects for the effect of heliocentric distance $R$, geocentric distance $\Delta$, and the detection efficiency. Doing so yields (Trujillo et al. 2001a)

$$
q=4.0_{-0.5}^{+0.6}(1 \sigma) .
$$

Table 2 shows that various survey results are in general agreement with each other. The Classical Belt thus contains $N(r>1 \mathrm{~km}) \sim 10^{10}, N(r>50 \mathrm{~km}) \sim 3 \times 10^{4}$, and $N(r>1000 \mathrm{~km}) \sim 10 \mathrm{KBOs}$. There is more than enough to supply the Jupiterfamily comets (see "Relationship to Other Solar System Bodies" below) (Levison

TABLE 2 Selected size distribution measurements of KBOs

\begin{tabular}{llll}
\hline $\begin{array}{l}\text { Discovery } \\
\boldsymbol{m}_{\boldsymbol{R}}\end{array}$ & $\begin{array}{l}\text { Number of KBOs } \\
\text { found }\end{array}$ & $\boldsymbol{q}$ & Reference \\
\hline $21.1-24.6$ & 86 & $4.0_{-0.5}^{+0.6}$ & Trujillo et al. (2001a) \\
$23.4-26.0$ & 17 & $4.4 \pm 0.3$ & Gladman et al. (2001) \\
$25.5-27.2^{\mathrm{a}}$ & 2 & $3.6 \pm 0.1$ & Chiang \& Brown (1999) \\
$23.8-26.7$ & 6 & $3.7 \pm 0.2$ & Luu \& Jewitt $(1998)^{\mathrm{b}}$ \\
$23.0-25.8$ & 5 & $4.8_{-0.6}^{+0.5}$ & Gladman et al. (1998) \\
$20.6-23.0$ & 13 & $4.0 \pm 0.5$ & Jewitt et al. $(1998)^{\mathrm{b}}$ \\
\hline${ }^{\mathrm{a}} \mathrm{V}$ magnitude. & \multicolumn{3}{l}{} \\
${ }^{\mathrm{b}}$ Extrapolated from CLF slope $\alpha$, according to $q=5 \alpha+1$. &
\end{tabular}


\& Duncan 1994, Duncan \& Levison 1997), and up to 10 Pluto-size bodies - all, presumably, much more distant than Pluto.

\section{Total Mass}

The total mass of the Kuiper Belt can be estimated by integrating over the size distribution, between a minimum radius $r_{\text {min }}$ and a maximum radius $r_{\text {max }}$. Current observations are far from probing the small end of the Kuiper Belt size distribution, so $r_{\text {min }}$ remains unconstrained, but the best-fitting $r_{\text {max }}$ can be determined by fitting Monte Carlo simulations of the KBO population to the data. Existing data rule out $r_{\text {max }} \leq 250 \mathrm{~km}$ at the $3 \sigma$ level, and can be fitted with $r_{\text {max }} \geq 500 \mathrm{~km}$ (Figure 8 , see Trujillo et al. 2001b). Formally, however, there is no evidence for a maximum-size

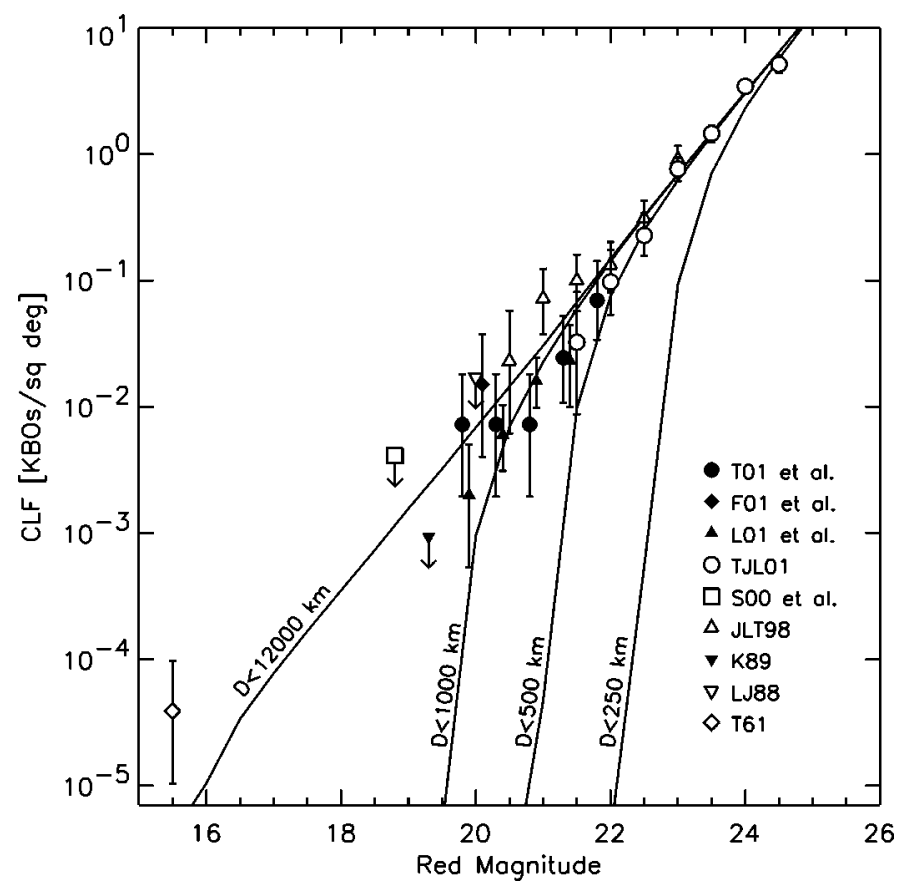

Figure 8 The observed cumulative luminosity function (CLF) for KBOs, adopting the $q=4.2$ size distribution. The legend is as follows: T01 et al. $=$ Trujillo et al. (2001b), F01 et al. = Ferrin et al. (2001), L01 et al. = Larsen et al. (2001), TJL01 = Trujillo et al. (2001a), S00 et al. = Sheppard et al. (2000) (upper limit), JLT98 = Jewitt et al. (1998), K89 = Kowal (1989) (upper limit), LJ88 = Luu \& Jewitt (1988) (upper limit), T61 = Tombaugh (1961). The lines correspond to model KBO populations with (left to right) $r_{\text {max }}=6000 \mathrm{~km}, 500 \mathrm{~km}, 250 \mathrm{~km}$, and $125 \mathrm{~km}$. The data rule out $r_{\text {max }} \leq 250$ $\mathrm{km}$, but cannot distinguish between $r_{\max }=500 \mathrm{~km}$ and larger. Figure adapted from Trujillo et al. (2001b). 
cutoff; if such a cutoff exists, it must be $\geq 1000 \mathrm{~km}$. We note that $r_{\max }=1000 \mathrm{~km}$ would be consistent with Pluto $(r=1100 \mathrm{~km})$ being the largest KBO known, but reiterate that a larger $r_{\max }$ cannot be ruled out.

The total mass of a $q=4$ distribution is

$$
M=\frac{4 \times 10^{9} \pi \rho \Gamma}{3}\left(\frac{0.04}{p_{R}}\right)^{3 / 2} \ln \left(\frac{r_{\max }}{r_{\min }}\right),
$$

where $\rho\left[\mathrm{kg} \mathrm{m}^{-3}\right]$ is the bulk density and $p_{R}$ is the red geometric albedo. The bulk density of KBOs is unknown, but can be guessed from related solar system objects. KBOs are believed to be precursors of short-period comets, whose nuclei have $\rho=500-1000 \mathrm{~kg} \mathrm{~m}^{-3}$ (Rickman et al. 1987). At the other end, the (gravitationally self-compressed) density of Pluto and Charon - the two largest known KBOs - is $\rho \sim 2000 \mathrm{~kg} \mathrm{~m}^{-3}$. Adopting the plausible values $r_{\min }=1 \mathrm{~km}, r_{\max }=1000 \mathrm{~km}, \rho=$ $1000 \mathrm{~kg} \mathrm{~m}^{-3}$ (Jewitt \& Sheppard 2002) albedo $p_{R}=0.07$ (as measured for the KBO 20,000 Varuna, Jewitt et al. 2001; see "Albedos" below) yields a total mass $M=0.08 M_{\oplus}$.

The 7\% albedo of 20,000 Varuna may or may not be representative of the KBO population. Size deduced from optical data scales as $p_{R}^{-1 / 2}$ and mass as $P_{R}^{-3 / 2}$, so higher albedos would lower the mass estimate above (for example, a factor-of-10 increase in the albedo results in a factor-of-30 decrease in mass). While we think it very likely that the KBO albedos are generally close to the $p_{R}=0.07$ assumed, it will be important to secure measurements of $p_{R}$ for a large sample of KBOs when possible [e.g., with the Space Infrared Telescope Facility (SIRTF)].

The optical mass is consistent with earlier dynamical limits of about $1 \mathrm{M}_{\oplus}$ (Hamid et al. 1968, Hogg et al. 1991), but is much smaller than would be expected from extrapolating the surface mass density of the solar system. In particular, this mass is far too small to form the larger KBOs within a $\sim 10^{8}$ year timescale imposed by the nearby accretion of Neptune (Lissauer et al. 1996) and implies a much more massive original Kuiper Belt (see "Formation of the Kuiper Belt" above). Accretion models predict that the original Kuiper Belt was roughly 100 times more massive than the current Belt (Kenyon \& Luu 1999a). Scattering by Neptune may have helped to deplete the original Belt, but could not be the major culprit (Holman \& Wisdom 1993). Collisional grinding might have been an effective mass loss mechanism (Stern 1996, Kenyon \& Luu 1999a). However, KBOs larger than $\sim 100$ $\mathrm{km}$ are largely immune to collisional disruption, and should be original remnants from the earliest days of the solar system.

\section{RADIAL EXTENT OF THE CLASSICAL KUIPER BELT}

The apparent brightness of a given KBO varies as $R^{-4}$, where $R$ is the heliocentric distance, imposing a daunting bias against the detection of very distant KBOs. Nevertheless, several independent survey results are consistent with the KBO surface density of Classical KBOs dropping steeply near 50 AU (Jewitt et al. 1996, 
1998; Dones 1997; Allen et al. 2001; Trujillo et al. 2001a). Trujillo \& Brown (2001) narrowed the location of the edge to $47 \pm 1 \mathrm{AU}$, assuming a differential size distribution with power law index $q=4$ and geometric albedo 0.04. This edge persists even for other size distributions $(3<q<5)$ and other albedo assumptions. Gladman et al. (1998) argued that a steep power law size distribution could produce an apparent outer edge to the belt, but the data already showed that sufficiently steep distributions $(q \sim 5)$ were not viable (Table 2$)$. Gladman et al. (2001) also cited recent discoveries of KBOs beyond 48 AU to argue against the density drop-off distances, although they themselves admitted that it was not clear whether these distant KBOs were members of the Classical Kuiper Belt. In short, the reliable data convincingly show that $\sim 50$ AU marks the edge of the Classical Kuiper Belt. (The SKBOs are excluded, even though they have large $e$ 's and $a$ 's that carry them as far as several hundred AUs at aphelion.)

What could be the cause of this unexpected outer boundary to the Classical Kuiper Belt? Suggested explanations include (Jewitt et al. 1998)

- systematically lower albedos of KBOs beyond $50 \mathrm{AU}$, rendering more distant objects harder to detect. This explanation is ad hoc, and has no clear physical foundation. Indeed, one might naively expect that the albedos of more distant KBOs of a given size should be higher, owing to the increased stability of ice at larger distances and lower temperatures.

- a decrease in the maximum size of KBOs $\left(r_{\max }\right)$ with increasing semimajor axis. Since all published surveys are magnitude-limited, only the largest KBOs can be detected at larger distances, and a decrease in $r_{\max }$ with increasing semi-major axis would give rise to an apparent edge to the KBO distribution. The rate of growth of the largest body of mass $M$ in a swarm of planetesimals is (Wetherill 1980):

$$
\frac{d M}{d t}=\pi r^{2} \rho_{0} v(1+2 \theta)
$$

where $\rho_{0}$ is the mean density of matter in the swarm and $v$ is the mean velocity of the swarm. The term $(1+2 \theta)$ represents the gravitational enhancement of the collisional cross section:

$$
(1+2 \theta)=1+\left(v_{e}^{2} / v^{2}\right),
$$

where $v_{e}$ is the escape velocity; $\theta$ is often called the Safronov number and has values 2-5 for most situations in planetary growth (Safronov 1969). In terms of the body's radius $r$ and density $\rho$, Equation 5 becomes

$$
\frac{d r}{d t}=\frac{\rho_{0} v}{4 \rho}(1+2 \theta) \text {. }
$$

The swarm density $\rho_{0}$ is related to the surface density $\sigma_{0}$ by (Safronov 1969)

$$
\rho_{0}=\frac{4 \sigma_{0}}{v P},
$$


where $P$ is the body's orbital period. In a nebula whose surface density $\sigma_{0}$ scales with distance as $R^{-2}$, the growth timescale $\tau$ is thus

$$
\tau=r / \dot{r} \propto \frac{r \rho R^{3.5}}{(1+2 \theta)} .
$$

In a given time, the size of the largest objects grown by accretion thus should scale as $R^{-3.5}$. All other things being equal, the largest objects at $50 \mathrm{AU}$ should be smaller than those at $40 \mathrm{AU}$ by a factor $(40 / 50)^{3.5}=0.46$. This is too small a factor to account for the deficiency of objects discovered at $R \geq 50 \mathrm{AU}$. So, while the sense of this effect is correct, the magnitude appears too small to account for the observations. In support of this conclusion we note that there is no significant trend of maximum size with heliocentric distance across the inner Kuiper Belt (from $30 \mathrm{AU}$ to $50 \mathrm{AU}$ ).

- the steep density drop-off in the Kuiper Belt near 50 AU corresponding to a real edge. One possibility that has been numerically explored is tidal truncation by a passing star (Ida et al. 2000b). According to these simulations, a passing star with periapse at 150-200 AU would truncate the disk at $50 \mathrm{AU}$, as is observed. Ida et al. further claimed that the resonant KBOs at $39 \mathrm{AU}$ could survive such a stellar close encounter. The likelihood of a close stellar passage in the present environment of the Sun is extremely remote, but if the Sun were born in a cluster, the high stellar densities and short encounter times might render this scenario more plausible (Eggers et al. 1997, Fernandez \& Brunini 2000). We note that the smallest measured Orion proplyds have sizes comparable to the inferred diameter of the KBO belt, perhaps because they were tidally truncated (McCaughrean \& O'Dell 1996). The contamination of the protoplanetary disk by ejecta from nearby exploding supernovae provides independent evidence that the Sun was born in a cluster containing perhaps 2000 stars (Adams \& Laughlin 2001).

\section{PHYSICAL PROPERTIES}

\section{Albedos}

The only reliable KBO albedos available are those of Pluto (0.5-0.7, Tholen \& Buie 1997), Charon ( 0.4, Tholen \& Buie 1997), and 20,000 Varuna (0.07, Jewitt et al. 2001). The large albedo of Pluto is produced by the interaction between its surface and its atmosphere, whereby gases in the atmosphere condense onto the surface as frost and give rise to a large albedo. By that argument, the albedos of those KBOs too small to sustain atmospheres are not expected to be high. This is supported by indirect evidence provided by the Centaurs and the known comet nuclei (Figure 9). Centaurs are objects on unstable, planet-crossing orbits between Jupiter and Neptune, and their transient nature suggests that they are ex-KBOs that have been scattered out of the Kuiper Belt (see "Relationship to Other Solar 


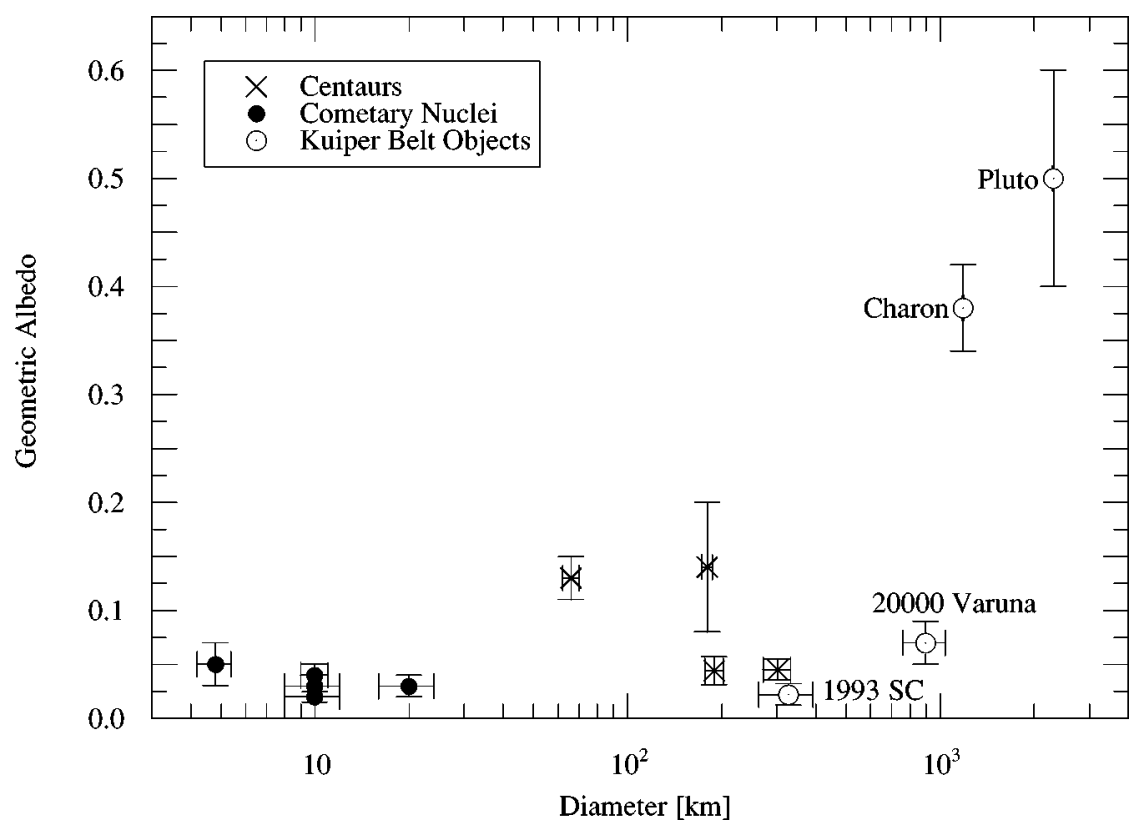

Figure 9 Plot of albedos vs. diameter for comet nuclei, Centaurs, Pluto-Charon, and the KBOs 20000 Varuna and 1993 SC. Figure adapted from Jewitt et al. (2001).

System Bodies" below). Figure 9 suggests a trend towards higher albedos at larger sizes: The small cometary nuclei are the darkest, whereas Pluto and Charon are the most reflective. Varuna, with a radius $40 \%$ that of Pluto, lies between these two extremes because its gravity is insufficient to retain an atmosphere. However, given the fact that water ice has been detected on several KBOs (see "Spectral Properties" below), a patchy frost distribution on the surface of Varuna cannot be excluded. An initial search for $\mathrm{CO}$ outgassing from KBOs has proved negative (Bockelee-Morvan et al. 2001).

\section{Colors}

The colors of KBOs provide a first-order measure of surface composition, albeit compounded with effects caused by size-dependent scattering from regolith particles (Moroz et al. 1998). The optical colors are the most easily measured: colors of about 3 dozen KBOs are available (Luu \& Jewitt 1996; Tegler \& Romanishin 1998, 2000; Green et al. 1997; Barucci et al. 2000; Jewitt \& Luu 2001). Figure 10 shows that $\mathrm{KBO}$ colors are widely distributed, with $0.7 \leq \mathrm{B}-\mathrm{V} \leq 1.2$ and $0.4 \leq \mathrm{V}-\mathrm{R} \leq 0.8$ (for comparison, solar colors are $\mathrm{B}-\mathrm{V}=0.67$, and $\mathrm{V}-\mathrm{R}=0.36)$. The $\mathrm{B}-\mathrm{V}, \mathrm{V}-\mathrm{R}$, and $\mathrm{R}-\mathrm{I}$ colors are strongly correlated in a statistical sense: Objects that are red at $4500 \AA$ are also red at $8000 \AA$. J-band photometry 


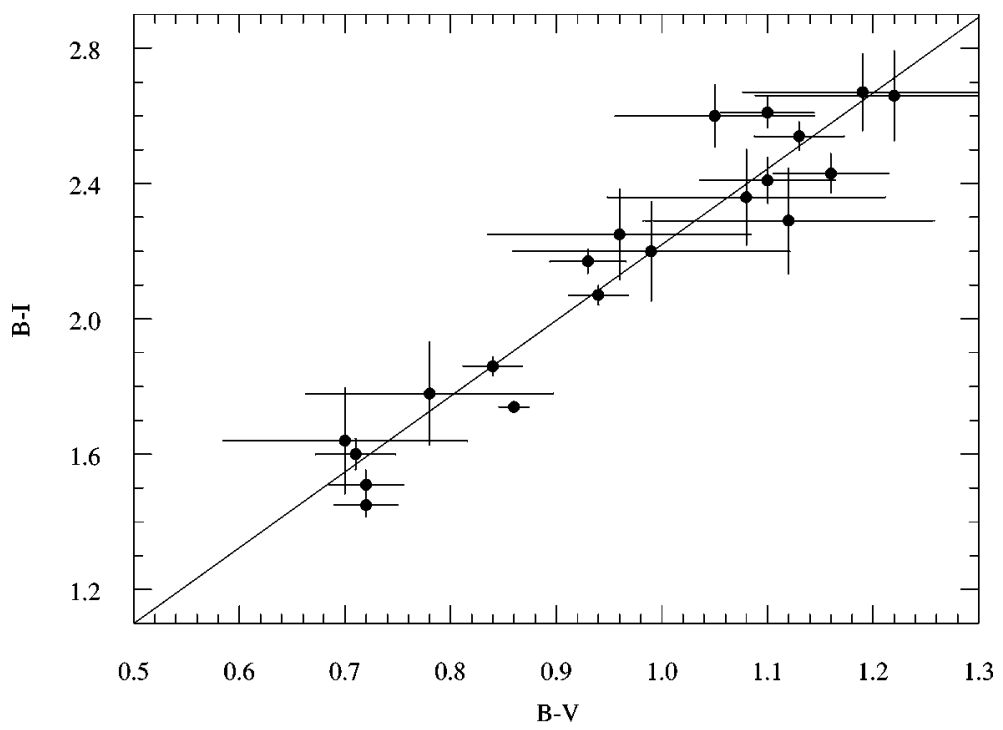

Figure 10 B-V vs. B-I for KBOs (with $\sigma_{B-V}<0.15$ mag) observed by Jewitt \& Luu (2001). The straight line is a least-squares fit to guide the eye. Figure from Jewitt \& Luu (2001).

available is also available for a dozen KBOs, showing that this correlation extends into the infrared, as can be seen in Figure 11. These correlations suggest that a single agent is responsible for the reddened colors from optical to infrared wavelengths; this property may help identify the agent among the low-albedo minerals with similar colors.

The color diversity of the KBOs (Luu \& Jewitt 1996) came as a surprise since it was expected that all KBOs should suffer from long-term irradiation, and should all sport irradiation mantles (e.g., Strazzulla \& Johnson 1991). When carboncontaining ices are irradiated by high energy particles prevalent in interplanetary space such as cosmic rays and solar wind particles, a solid residue is created that is rich in complex carbon compounds and reddish in color. The penetration depths of $\mathrm{GeV}$ protons in solid ice are near $1 \mathrm{~m}$ (Strazzulla \& Johnson 1991). An irradiation mantle of this thickness should grow on timescales $\sim 10^{8 \pm 1} \mathrm{yr}$ (Shul'man 1972). After 4.5 billion years, all KBOs are thus expected to sport a red, nonvolatile, organic-rich crust, but this is clearly not the case. Could different dynamics (see "Structure of the Kuiper Belt" above) or physical properties be responsible for the color diversity? Jewitt \& Luu (2001) searched for and found no correlation between the optical colors and absolute magnitude or any orbital parameter (heliocentric distance, semimajor axis, etc.).

The origin of color diversity could reflect intrinsic compositional differences among the KBOs, or it could be a result of collisional resurfacing (Luu \& Jewitt 


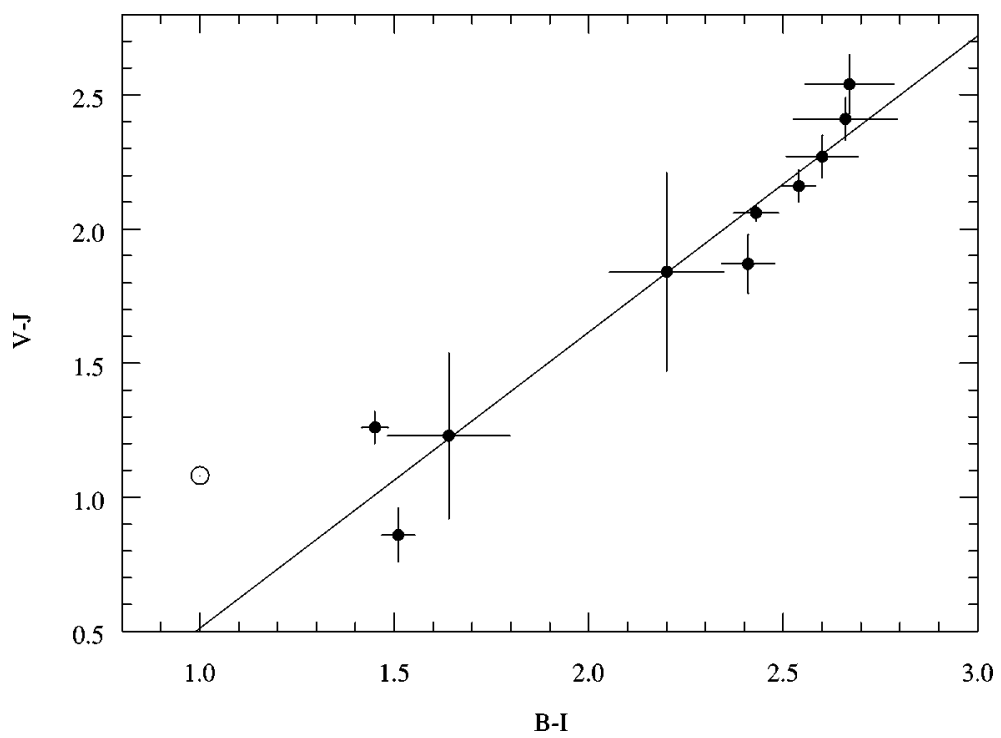

Figure 11 B-I vs. V-J for KBOs observed by Jewitt \& Luu (2001) and Davies et al. (2000). The straight line is a least-squares fit to guide the eye. The color of the Sun is marked. Figure from Jewitt \& Luu (2001).

1996). In the latter case, the color diversity would result from competition between two counteracting processes: irradiation reddening and collisional resurfacing. Occasional strong collisions may shower the surface with impact debris which would be unirradiated and possibly ice-rich. If this freshly excavated material were of a different composition and color from the irradiation mantle, the net result would be a resetting of the color by each large impact. Depending on how much of the surface is covered with impact debris, resurfacing may change the overall surface color. However, this resurfacing model works well only when $(a)$ the irradiated and buried materials have quite different colors, and $(b)$ the timescale for collisional resurfacing is comparable to the timescale for irradiation damage of the exposed surface material.

The resurfacing scenario makes several predictions. First, since the collision timescale depends on the cross-section of a $\mathrm{KBO}$, the globally averaged color should also be related to KBO size. This is not observed in the currently available data. Second, many KBOs should display rotational color/compositional variations, due to incomplete blanketing of their surfaces by impact excavated matter. However, the general agreement between colors measured by different investigators at random rotational phases suggests that color anisotropy is rare (see Table 5 of Jewitt \& Luu 2001). Detailed measurements of Centaur 8405 Asbolus (Kern et al. 2000), on the other hand, show evidence for azimuthal compositional variations that are consistent with resurfacing. Recently, Trujillo \& Brown (2002a) 
reported a significant correlation between color and inclination among the Classical KBOs but not among the Plutinos. No correlation was found between color and any other orbital parameter. A fair statement would be that, although the resurfacing hypothesis is not yet ruled out by the available data, there is presently no compelling support for it. The correct explanation for the KBO color distribution may involve a complex combination of both evolutionary and cosmogonic effects.

Tegler \& Romanishin $(1998,2000)$ have reported that the distribution of KBO colors was bimodal. If correct, this finding would have important implications for the origin of color diversity in the Kuiper Belt. However, bimodality has not been independently confirmed (Green et al. 1997, Barucci et al. 2000, Davies et al. 2000), and was not borne out by statistical tests applied to Tegler \& Romanishin's 1998 and 2000 data sets (either separately or combined, Jewitt \& Luu 2001). One can thus conclude that evidence for a bimodal color distribution is lacking, and that initial indications to the contrary result from a pathologically small sample.

The V-R color histograms of the KBOs are compared with those of other small solar system bodies in Figure 12. The KBOs have a large color range that is matched only by the Centaurs, which are believed to have originated in the Kuiper Belt (see "Relationship to Other Solar System Bodies" below). In particular, the most extreme red colors $(V-R>0.7)$ belong to the KBOs and the Centaurs. The facts that KBOs and Centaurs have similar color distributions, and that their color distributions are distinct from those of known asteroids and comet nuclei, suggest 1. a common origin for KBOs and Centaurs, and 2. the presence of indigenous primitive material on the surface of KBOs and Centaurs that may correspond to the predicted irradiation mantle (Luu \& Jewitt 1996). As shown in laboratory experiments, the mantle thickness depends on the actual irradiation flux and may be sufficiently thick $(\sim 1 \mathrm{~m})$ to survive a Centaur's first entry into the inner solar system, but this primitive surface is gradually lost/modified once KBOs leave the Kuiper Belt and join the comet population (Jewitt 2002). It is of interest that the extreme red color as well as the near-IR spectrum of the Centaur 5145 Pholus have been reproduced by complex hydrocarbons (Wilson et al. 1994, Cruikshank et al. 1998).

\section{Spectral Properties}

Few high quality spectra of KBOs exist and even fewer spectral features have been reported. KBOs with reported features include $1996 \mathrm{TO}_{66}$ (Brown et al. 1999), 20,000 Varuna (Licandro et al. 2001), and 1999 DE$_{9}$ (Jewitt \& Luu 2001). The first two show the familiar 1.5 and $2 \mu \mathrm{m}$ features of water ice, but the $\mathrm{DE}_{9}$ spectrum appears more complex. Figure 13 shows the $1999 \mathrm{DE}_{9}$ spectrum, with apparent absorption features near $1.4 \mu \mathrm{m}, 1.6 \mu \mathrm{m}$, and $2 \mu \mathrm{m}$, and a weak absorption feature at $\sim 2.25 \mu \mathrm{m}$. The reflectivity also drops shortward of $1.4 \mu \mathrm{m}$ down to the limit of the spectral coverage at $1.0 \mu \mathrm{m}$. Water ice has well-known diagnostic features at $1.55 \mu \mathrm{m}, 1.65 \mu \mathrm{m}$, and $2.02 \mu \mathrm{m}$ (Clark 1981a), and in the case of fine grain frost, the $1.55 \mu \mathrm{m}$ and $1.65 \mu \mathrm{m}$ features become blended and weaker 


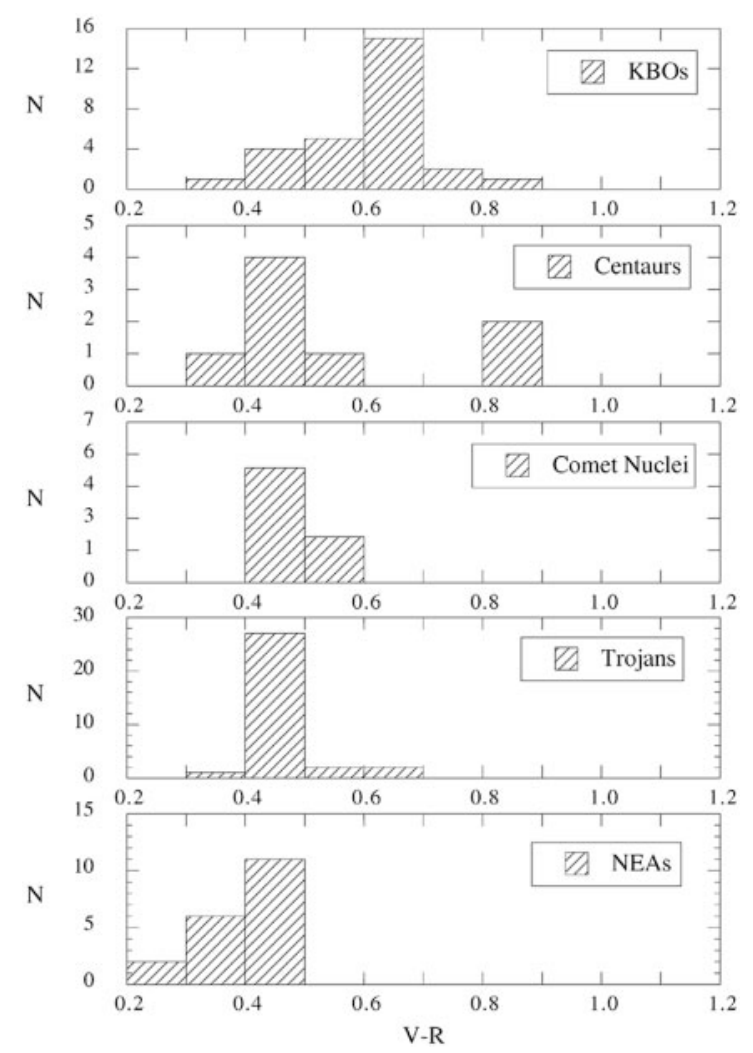

Figure 12 Comparison of Kuiper Belt V-R colors with those of other small solar system bodies: near-Earth asteroids (NEAs), Trojan asteroids, comet nuclei. Figure adapted from Luu \& Jewitt (1996).

than the $2 \mu \mathrm{m}$ feature (Clark 1981a), much like the $\mathrm{DE}_{9}$ spectrum. The water ice high-overtone band at $1.25 \mu \mathrm{m}$ is not seen in the $\mathrm{DE}_{9}$ spectrum, and this is possibly a consequence of the presence of other minerals in addition to water ice (Clark 1981a). The weakness of the water ice features suggests a low abundance of water; the $\mathrm{DE}_{9}$ spectrum is very similar to that of a mixture of Mauna Kea red cinder and 1\% water ice (Clark 1981b), also shown in Figure 13.

The features at $1.4 \mu \mathrm{m}$ and $2.25 \mu \mathrm{m}$ are usually diagnostic of the presence of the $\mathrm{OH}$ stretch (Hunt 1977), and the exact location of the $2.25 \mu \mathrm{m}$ feature can be diagnostic of the compound (either $\mathrm{Al}$ or $\mathrm{Mg}$ ) associated with the $\mathrm{OH}$ stretch. However, the quality of the $\mathrm{DE}_{9}$ spectrum is not good enough for this identification. The spectral drop toward $1 \mu \mathrm{m}$ may be indicative of an absorption feature, more specifically, the $1 \mu \mathrm{m}$ feature due to the ferrous ion $\left(\mathrm{Fe}^{2+}\right)$ in sixfold coordination, a feature well known from spectra of silicates such as olivine and 


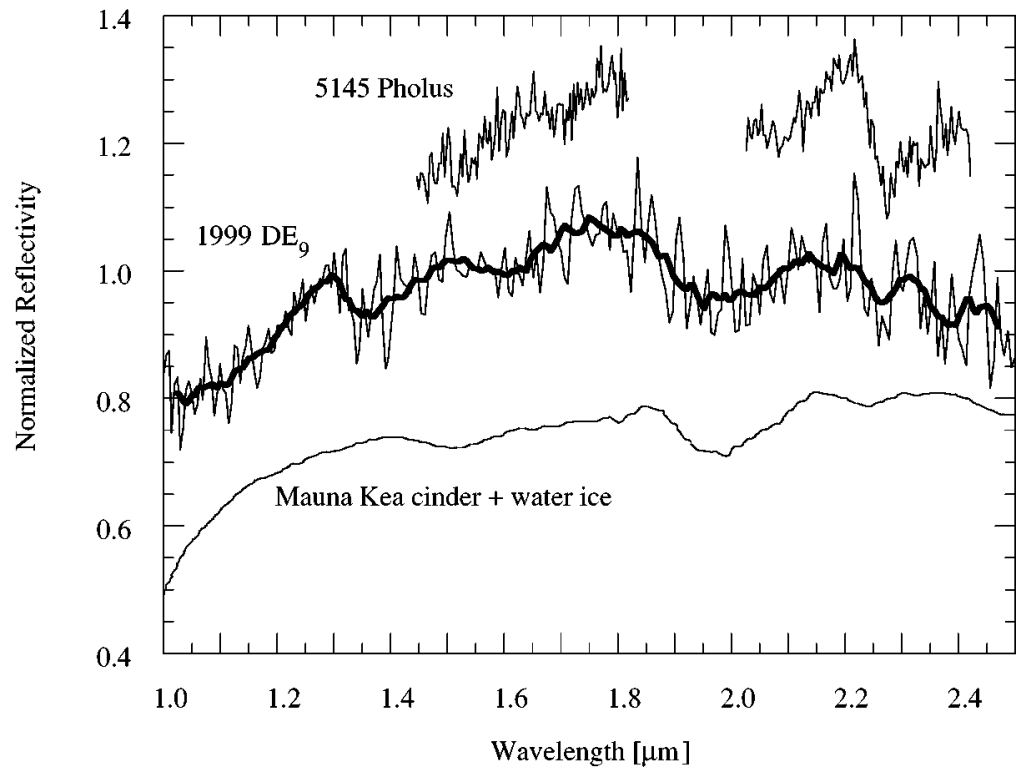

Figure 13 Reflection spectrum of KBO $1999 \mathrm{DE}_{9}$, compared with Mauka Kea cinder $+\mathrm{H}_{2} \mathrm{O}$ ice, and the Centaur 5145 Pholus. The thick line through $\mathrm{DE}_{9}$ is a running mean added to guide the eye. $\mathrm{DE}_{9}$ shows absorption features due to water ice $(2 \mu \mathrm{m})$, silicates $(1 \mu \mathrm{m})$, and the $\mathrm{OH}$ stretch $(1.4$ and $2.25 \mu \mathrm{m})$. Figure adapted from Jewitt \& Luu (2001).

pyroxene (Hunt 1977). We rule out pyroxene as a possible match for $\mathrm{DE}_{9}$ because pyroxene usually shows a strong feature near $1.8 \mu \mathrm{m}$ which is not seen in $\mathrm{DE}_{9}$. The olivine forsterite $\left(\mathrm{Mg}_{2} \mathrm{SiO}_{4}\right)$ has been found in comets (Crovisier et al. 2000), and would fit the $1 \mu \mathrm{m}$ absorption in DE9's spectrum (Figure 13), if the feature is confirmed.

Other remarkably featureless KBO spectra exist, e.g., $2000 \mathrm{~EB}_{173}$ (Brown et al. 2000), 1996 TL66 (Jewitt \& Luu 1998), and 1993 SC (Luu \& Jewitt 1996). Based on this handful of results, it appears that KBO spectra (thus far) may be roughly divided into at least two groups: one characterized by nearly featureless neutral/bluish spectra, and the other by spectral features indicating the presence of water ice and/or hydroxyl. Brown et al. (2000) attempt to link the presence of the water ice features to the albedo, but this is probably overly naive since laboratory experiments have indicated that water ice features can be very apparent even when the albedo is low (Clark 1981b). Luu et al. (2000) speculate that water ice is ubiquitous on KBOs but may not always be detectable due to its low abundance or high degree of contamination. The spectral diversity exhibited by the few well observed KBOs thus seems to mimic the optical color diversity. 


\section{Rotation}

The rotation states of KBOs, like those of asteroids, can be determined from timeresolved measurements of the scattered light. The larger KBOs are thought to be primary products of accretion and, as such, may preserve the angular momentum they acquired at formation. Measurements of the distribution of angular momenta are thus of great interest as a potential constraint on the physics of accretion. By contrast, the smaller KBOs and almost all main-belt asteroids have been heavily processed by collisions, and their current spin states and angular momenta are unlikely to be primordial.

Unfortunately, the rotational states of most KBOs are, as yet, poorly defined. Romanishin \& Tegler (1999) reported lightcurve variations for $1995 \mathrm{QY}_{9}, 1994$ $\mathrm{VK}_{8}$, and $1994 \mathrm{~TB}$, and reported a correlation between lightcurve amplitude and absolute magnitude (a proxy for size). However, Collander-Brown et al. (1999) independently observed $1994 \mathrm{VK}_{8}$ but detected no clear rotational signature. Hainaut et al. (2000) reported a $6.25 \mathrm{hr}$ periodicity in $1996 \mathrm{TO}_{66}$ and an amplitude varying from 0.12 to $0.33 \mathrm{mag}$, but the latter result is contradicted by Romanishin \& Tegler (1999) who set a 0.1 mag upper limit to the amplitude. One of the few convincing and remarkable lightcurves is that of $(20,000)$ Varuna (Figure 14), which has a double-peak period of $6.34 \mathrm{hr}$ and amplitude 0.42 mag (Jewitt \& Sheppard 2002). The rapid rotation and large photometric range of Varuna suggests a body

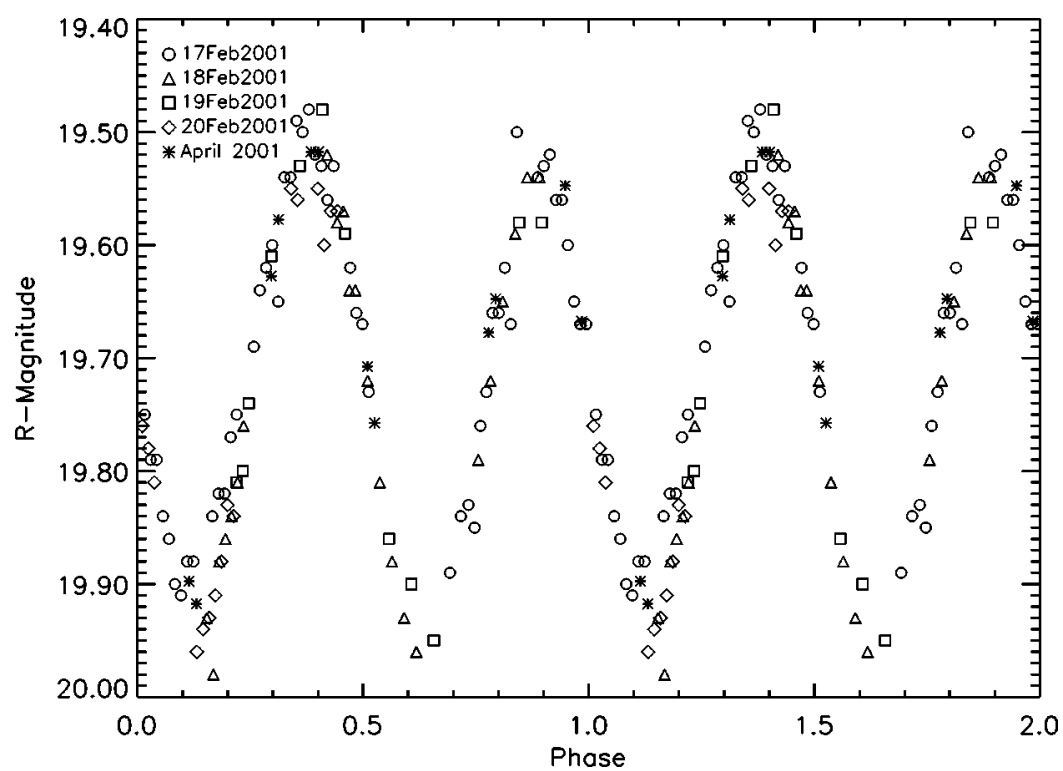

Figure 14 Phased lightcurve (in R band) of KBO (20000) Varuna, assuming a double-peak rotation period $P_{\text {rot }}=6.3436$ hours. Error bars are 0.02 mag (not plotted for the sake of clarity). Figure from Jewitt \& Sheppard (2002). 
that is rotationally distorted/deformed from spherical shape by its own specific angular momentum. This object, like most large main-belt asteroids, is probably structurally weak as a result of repeated energetic impacts. The resulting rubble pile structure enables Varuna to adopt a near-equilibrium form. Modelled as an equilibrium rotational figure (a Jacobi ellipsoid), Varuna is found to have a bulk density $\rho \geq 1000 \mathrm{~kg} \mathrm{~m}^{-3}$ (Jewitt \& Sheppard 2002). This low density suggests that Varuna must be porous (at the 5-30\% level) in order to allow for a cosmochemically normal admixture of refractory compounds (rocks). Many of the icy satellites of Saturn and Uranus also possess densities near that of water ice: They may also be porous (Kossacki \& Leliwa-Kopystynski 1993).

\section{INTERNAL STRUCTURE}

It is likely that, even though they are ice-rich, the KBOs contain a significant or even dominant component of refractory material incorporated from the dusty protosolar disk. Measurements of the outgassed products of near-sun comets yield dust/gas production rate ratios, $F \gtrsim 1$ (Lisse et al. 1998, Jewitt \& Matthews 1999), which may be indicative of the volatile fraction in KBOs. In any case, the refractories will include radioactive nuclei $(\mathrm{K}, \mathrm{Th}, \mathrm{U})$ whose decay constitutes a source of internal heat. In small KBOs, this heat can be conducted to the surface and lost to space by radiation. The larger KBOs, however, cannot conduct radiogenic heat on timescales comparable to their age. Therefore, we expect that the larger KBOs should be internally heated, perhaps leading to partial differentiation, mobilization of internal gases, outgassing, and even cryogenic volcanism.

The dividing line between objects that are able to cool conductively and those that are too large to lose radiogenic heat occurs when the conduction cooling time, $\tau=r^{2} / \kappa$, equals the age of the solar system, $t_{S S}$. This occurs for critical radius

$$
r=\sqrt{\kappa t_{S S}}
$$

For the thermal diffusivity of solid ice, $\kappa=10^{-6} \mathrm{~m}^{2} \mathrm{~s}^{-1}$, and $t_{S S}=4.6 \mathrm{Gyr}=$ $3 \times 10^{17} \mathrm{~s}$, Equation 10 gives $r \sim 500 \mathrm{~km}$. For diffusivity appropriate to slightly porous ice, $\kappa \sim 10^{-7} \mathrm{~m}^{2} \mathrm{~s}^{-1}$, the critical radius is near $100 \mathrm{~km}$. Approximately $5000 \mathrm{KBOs}$ are larger than $100 \mathrm{~km}$ in radius. All these objects will be internally heated by radioactive decay, and the magnitude of the heating can be estimated by comparing the radioactive energy liberated over $t_{S S}$ with the heat capacity of the KBO. The specific energy generation due to radioactivity in chondritic material is $H \sim 10^{-12} \mathrm{~W} \mathrm{~kg}^{-1}$ (Stacey 1969). In KBOs, the heating rate will be diluted by mixing with (nonradioactive) ice, so we adopt $H=5 \times 10^{-13} \mathrm{~W} \mathrm{~kg}^{-1}$ to be conservative. For a body at the critical radius (Equation 10) the globally averaged temperature rise is of order

$$
\Delta T=\frac{H t_{S S}}{c_{P}},
$$


where $c_{P}\left[\mathrm{~J} \mathrm{~kg}^{-1} \mathrm{~K}^{-1}\right]$ is the specific heat capacity. With $c_{P} \sim 10^{3} \mathrm{~J} \mathrm{~kg}^{-1} \mathrm{~K}^{-1}$, we obtain $\Delta T \approx 150 \mathrm{~K}$. This is too small to melt water ice but sufficient to mobilize supervolatiles such as $\mathrm{CO}$ and $\mathrm{N}_{2}$, and to initiate crystalline phase transitions in water ice. We would thus expect KBO interiors to have a layered structure, with the more volatile ices concentrated outside the warm core.

Real KBO interiors are likely to be much more complex than implied by the simple calculation above. The complete collisional history of the Kuiper Belt, particularly towards the end of the $100 \mathrm{Myr}$ accretion period during which the density of objects was $\sim 100$ times larger than now (Kenyon \& Luu 1999a), may have produced a rubble-pile structure in many bodies. Internal void space may be common (Jewitt \& Sheppard 2002) and could have significant impact on the thermal and mechanical properties of KBOs. Heat transfer within a porous, volatile body can produce startling structural complexity (Prialnick 2000). De Sanctis et al. (2001) investigated the internal evolution of KBOs under the combined effect of radiogenic heating and solar irradiation, and found several possible outcomes. For example, KBOs could be very differentiated: The top several hundred meters are depleted in the most volatile ices (e.g., CO), followed by interlaced layers of depleted and enriched volatiles produced by the sublimation of supervolatiles from the inner regions and the recondensation of these volatiles in cooler layers. Alternatively, KBOs could also preserve undifferentiated cores, depending on the kind and amount of radiogenic elements present. The particular outcome depends strongly on the particular radiogenic material and the assumed physical parameters of the KBO (e.g., thermal conductivity, porosity, radius).

\section{BINARY KBOs}

Toth (1999) briefly speculated on the observability of KBO satellites, but the existence of binary KBOs (other than Pluto-Charon) was first established by Veillet et al. (2000). At the time of writing, 5 binary KBOs are known: Pluto-Charon (Tholen \& Buie 1997), 1998 WW $_{31}$ (Veillet et al. 2001), 2001 QT $_{297}$ (Elliot et al. 2001), $2001 \mathrm{QW}_{322}$ (Kavelaars et al. 2001), and $1999 \mathrm{TC}_{36}$ (Trujillo \& Brown 2002b). $\mathrm{TC}_{36}$ is the first binary trans-Neptunian that has been discovered in 3:2 mean-motion resonance with Neptune since Pluto-Charon. Images from the Hubble Space Telescope clearly show $1999 \mathrm{TC}_{36}$ to be accompanied by a secondary, separated by $\sim 0.37$ arcsec (Figure 15), implying an orbital distance of $\geq 8,000 \mathrm{~km}$. The detection of binary KBOs is important because such a configuration potentially allows the direct determination of the masses of the components.

The Kuiper Belt binaries are remarkable for their wide separations. For example, the KBO binary $2001 \mathrm{QW}_{322}$ has a separation near $100,000 \mathrm{~km}$, while the components are probably not much larger than $200 \mathrm{~km}$ in diameter (based on their absolute magnitude). The stability of such wide binaries is partly a result of the large scale of the respective Hill spheres of the KBOs. Long-term stability also places constraints on the mutual perturbation rate amongst KBOs. 
The formation of the binaries remains mysterious. The best characterized pair, Pluto-Charon, possesses a combined angular momentum per unit mass that is larger than the maximum possible for a stable, self-gravitating, strengthless body (McKinnon 1989). As a result, it is widely supposed that Charon was formed when Pluto was struck by a massive interloper. Charon would have accreted from the fraction of the debris that remained trapped in bound orbits after the collision, in much the same way that the Earth's Moon is thought to have formed following an early collision. The angular momenta of the other Kuiper Belt binaries are, pending orbit determinations, unknown, and the formation mechanisms are essentially unconstrained.

\section{RELATIONSHIP TO OTHER SOLAR SYSTEM BODIES}

\section{Pluto-Charon and Triton}

Much of the interest in the Kuiper Belt stems from its link to a remarkably diverse group of objects with origin in the outer solar systerm. The most scientifically appropriate identification of Pluto-Charon as the two largest known Kuiper Belt objects has already been discussed (see "Binary KBOs" above). An origin in the Kuiper Belt can probably be extended also to the captured Neptunian satellite Triton, given that this object is a near-twin of Pluto in terms of bulk physical properties. The identification of these large outer solar system bodies as KBOs provides a simple and consistent framework for their formation in the outer solar system, ruling out more contrived theories for their origin (Lyttleton 1936, Harrington \& van Flandern 1979, Farinella et al. 1979, Woolfson 1999). In this picture, Pluto and Triton formed in the trans-Neptunian region and became two of its largest members. However, this framework does not solve the puzzle of how Triton was captured into a satellite orbit around Neptune. Suggested capture mechanisms include a collision with an original satellite (Goldreich et al. 1989), or gas drag in a proto-Neptunian nebula (McKinnon \& Leith 1995). Further understanding of the population and dynamics of the Kuiper Belt may shed light on Triton's dynamical history.

\section{Comets and Centaurs}

At the small end of the size distribution in the outer solar system are the Jupiterfamily comets (comets whose orbits cross or closely approach Jupiter's orbit and which are therefore strongly influenced by the planet), believed to have originated in the Kuiper Belt. The nuclei of the Jupiter-family comets have sublimationlimited lifetimes of $\sim 10^{4} \mathrm{yr}$ and dynamically limited lifetimes of $\sim 10^{5} \mathrm{yr}$ (due to ejection by the planets or impact with planets or the sun) (Levison \& Duncan 1994). To maintain a steady state population over longer timescales the Jupiter-family comets must be replenished from a more stable reservoir. Whereas it was previously thought that the Jupiter-family comets could be derived from the long-period 
(Oort Cloud) comets by gravitational capture by the gas giant planets, detailed calculations show this path to the Jupiter-family comets to be inefficient. Instead, it is more likely that the Jupiter-family comets are escaped KBOs that have been scattered inward by the planets to the vicinity of Earth (Fernandez 1980, Duncan et al. 1988). The instability that triggers the ejection of KBOs from long-lived orbits beyond Neptune may be either dynamical chaos (Holman \& Wisdom 1993, Levison \& Duncan 1994) or collisional scattering (Ip \& Fernandez 1997). Once Neptune-crossing, the timescale for transfer to the inner planetary region is $10^{7}$ to $10^{8}$ years (Hahn \& Bailey 1990, Dones et al. 1999).

If Jupiter-family comets originate in the Kuiper Belt and eventually end up with orbits not much larger than Jupiter's, one would expect to find cometsin-transition, i.e., those objects in the process of making the journey from the Kuiper Belt into the inner solar system. These transition objects have probably been found in the Centaurs. Roughly 25 Centaurs are known as of August 2001. Some Centaurs already show cometary activity: The well-studied Centaur 2060 Chiron possesses ice on its surface (Luu et al. 2000), and has displayed a persistent coma for roughly a decade (e.g., Hartmann et al. 1990, Luu \& Jewitt 1990, Meech \& Belton 1990, Marcialis \& Buratti 1993) (Figure 16). Others (like 5145 Pholus) lack coma, making the nucleus directly accessible for study and reducing the likelihood that outgassing has altered the surface properties from the pristine state. As noted in "Colors" above, broadband photometry and spectral observations of the brightest Centaurs show a wide diversity, from neutral/blue 2060 Chiron to ultra-red 5145 Pholus, much like the color diversity of the KBOs. This color diversity, plus evidence for spectral similarity between the Centaurs and KBOs (Figure 13), are consistent with an origin in the Kuiper Belt for the Centaurs.

Measurements of the physical properties of cometary nuclei are complicated by the presence of near-nucleus coma, and reliable data exist for only a few. The most consistent and distinguishing property is the geometric albedo, for which very low values $0.02 \leq p_{V} \leq 0.04$ have been measured in 15 well-measured comets (Figure 1 of Fernandez et al. 2001), including Halley [Figure 17a, and comet Borrelly (Figure 17b)]. Spatially resolved images of the nucleus of P/Halley (Figure 17a) show that outgassing is largely confined to collimated jets that occupy perhaps $10 \%$ of the nucleus surface. The remaining $90 \%$ is blanketed by dark, inert material that has been widely interpreted as a refractory mantle composed of fallback debris. The low albedo seen in both Halley and Borrelly suggests a carbon-rich composition, perhaps consisting of complex hydrocarbon polymers (Moroz et al. 1998). The measured short-period comet nuclei resemble elongated spheroids, with effective radii in the range $1 \leq r \leq 10 \mathrm{~km}$ (Jewitt 1990, Lamy et al. 2000) and axis ratios up to 2:1. Larger nuclei exist but are rare. Most comet nuclei are probably collision fragments, while KBOs larger than $100 \mathrm{~km}$ are likely to have survived collisional disruption (Farinella \& Davis 1996, Kenyon \& Luu 1999a). If so, the short-period comets may not be as primitive as originally believed. The form of the comet nucleus size distribution has not been accurately measured because of 
practical difficulties by coma contamination. Measurements of the size distribution of small impact craters on the surfaces of the satellites of the outer planets provide the best, although admittedly indirect, measure of the nucleus size distribution. These give a projectile differential size distribution index $q=3$ (McKinnon et al. 1991).

\section{FORMATION OF THE KUIPER BELT}

In all likelihood, the Kuiper Belt is a processed remnant of the protoplanetary disk, and therein lies its enormous scientific interest. Efforts to reconstruct the growth and dynamical evolution of the KBOs will probably be the most fruitful steps taken in the next decade toward understanding accretion in the solar system. The problem of the formation of the KBOs was tackled early on by Stern (1996) and Stern \& Colwell (1997), using the particle-in-a-box paradigm where the collisions of particles are treated in a statistical manner. These models allowed for the possibility of fragmentation during the accretion process, but did not fully model the velocity evolution of the KBOs. This omission is important because the distribution of planetesimal velocities plays a crucial role in determining the growth rate. Nevertheless, they recognized that the present mass of the Belt $\left(\sim 0.1 M_{\oplus}\right.$, see "Total Mass" above) was too small to build up the currently observed distribution via binary accretion.

\section{Kenyon and Luu Model}

Kenyon \& Luu (1998, 1999a) performed the most detailed simulations yet of the growth of KBOs, with the 1999 work following simultaneously the evolution of both planetesimal masses and velocities, and allowing for fragmentation during the accretion process. Their model is based closely on Earth-accretion models of Wetherill \& Stewart (1993), also adopting the particle-in-a-box approach (Safronov 1969). The calculations typically begin with an initial distribution of $\sim 100$ m planetesimals located in a single annulus around the Sun. As planetesimals collide with each other, they may grow larger due to accretion, smaller due to fragmentation, or become completely pulverized in a catastrophic collision. The mass distribution is recorded in $n$ mass bins, and the mass and velocity in each bin is recorded at each time step, after calculating the collision rates, the outcome of each collision, and the velocity changes due to collisions and long-range gravitational interactions. The calculations are usually carried out for $\sim 10^{8} \mathrm{yr}$, the expected formation timescale for Neptune (Lissauer et al. 1996), since a full grown Neptune is expected to excite the orbits of KBOs and thus inhibit further growth.

Adopting an annulus width $\Delta a=6 \mathrm{AU}$, centered at heliocentric distance $a=$ $35 \mathrm{AU}$, containing $80 \mathrm{~m}$ radius planetesimals with mass density $\rho_{0}=1500 \mathrm{~kg} \mathrm{~m}^{-3}$ and initial eccentricities $e_{0}=10^{-4}-10^{-2}$, the results can be summarized as follows: 
1. It is possible to form the observed trans-Neptunian distribution within a $10^{8}$-yr timescale with an initial annulus mass $M_{0}=3-30 M_{\oplus}$. This initial mass is 30 to 300 times larger than the current mass of the Kuiper Belt, but is roughly consistent with to the mass of the Minimum Mass Solar Nebula $\left(M_{M M S N}\right.$, Hayashi et al. 1985), which predicts $M_{M M S N}=7-15 M_{\oplus}$ in the 32-38 AU annulus.

2. The final size distribution is determined by two competing physical processes: growth by mergers and erosion by fragmentation. It can be described by a broken (differential) power law with two power indices: $q=3.5$, for $r \leq 0.1-1 \mathrm{~km}$ (representing the fragment regime, Dohnanyi 1969), and $q=3.75-4.25$ for $r>1-10 \mathrm{~km}$ (merger regime). Several Pluto-sized bodies are naturally produced on the $10^{8}-\mathrm{yr}$ timescale, depending on the initial mass.

3. Fragmentation limits the maximum size reached by KBOs: $r_{\max }$ ranges from $450 \mathrm{~km}$ to $3000 \mathrm{~km}$, depending the tensile strength (a low tensile strength facilitates fragmentation, inhibiting growth).

4. Due to fragmentation, most of the initial mass ends up in smaller $(0.1-10 \mathrm{~km})$ objects that can be collisionally depleted over the age of the solar system. The present Kuiper Belt contains only $1-2 \%$ of the initial mass.

The final results, especially the final size distribution, are surprisingly insensitive to most input parameters (initial size distribution, initial planetesimal size, etc.). A typical outcome of the calculations is shown in Figure 18.

Figure 19 compares the model luminosity functions of KBOs with observations: The left panel shows models with $e_{0}=10^{-3}$ and different $M_{0}$, whereas the right panel shows models with a MMSN $M_{0}$ with different $e_{0}$. The models were originally computed to fit the Kowal (1989) data point, which has recently been invalidated (Trujillo et al. 2001a), but the models fit the remaining observations very well. It should be noted that the KL99a model is over-simplified: For example, it does not allow for the migration of KBOs, and it does not fully account for the dynamical effects of the largest bodies upon the planetesimal swarm. Nevertheless, it provides a self-consistent model for accretion and fragmentation in the early Kuiper Belt, and makes several testable predictions.

\section{EVOLUTION OF THE KUIPER BELT}

The picture of the Kuiper Belt that has emerged from dynamical and accretion models is that of a heavily attenuated population, eroded dynamically and physically by a variety of mass loss mechanisms. Long-term gravitational effects of the planets and overlapping secular resonances induce instabilities in large regions interior to $42 \mathrm{AU}$ (Figure 20). The Figure shows the good agreement between the dynamical calculations and the observed orbital distribution of KBOs: Most known KBOs are indeed located in stable regions. The current Kuiper Belt 

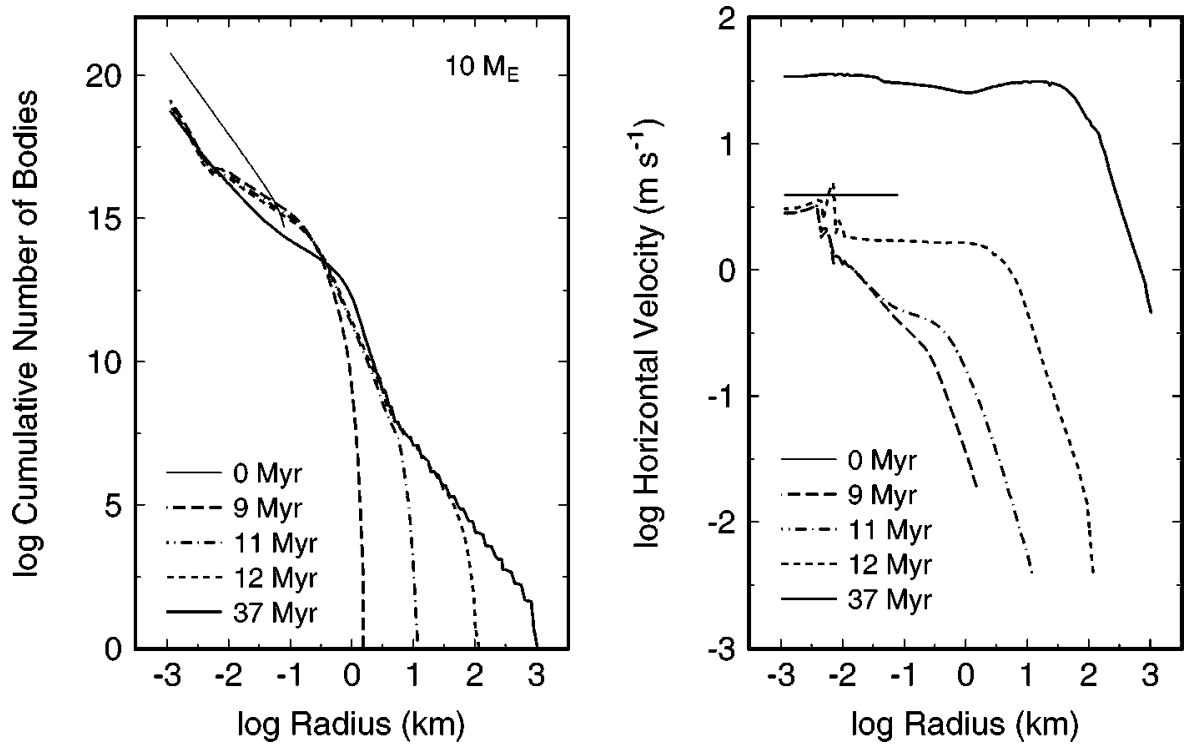

Figure 18 Size and velocity distributions for a model with initial mass $M_{0}=10 M_{\oplus}$, initial eccentricity $e_{0}=10^{-3}$, tensile strength $S_{0}=2 \times 10^{6} \mathrm{ergs} \mathrm{g}^{-1}$. Left, cumulative size distribution; right, horizontal velocity as a function of time. Figure adapted from Kenyon \& Luu (1999a).

contains far too little mass to form the observed population in the $\sim 10^{8} \mathrm{yr}$ available prior to the disruptive emergence of Neptune; instead, the original mass must have been $\sim 100$ times the current mass. Much of the original mass was ground to dust by collisions, then lost from the system (see "Kenyon and Luu Model" above). However, KBOs larger than $\sim 100 \mathrm{~km}$ are largely immune to collisional disruption (Farinella \& Davis 1996); they are thus likely to be true survivors from the earliest days of the solar system.

Other more violent mass loss mechanisms may have also occurred: Morbidelli \& Valsecchi (1997) and Petit et al. (1999) showed that Mars-sized bodies in eccentric orbits in the Kuiper Belt could excite the $e$ 's and $i$ 's of primordial KBOs to the current observed values. Yet another line of thought attributes the excited orbits of the Kuiper Belt to the birth environment of the Sun. Pointing out that stars are generally born in clusters and stay there for $\sim 10^{8}$ yr (Kroupa 1995, 1998), Ida et al. (2000b) proposed close stellar encounters as the mechanism to excite the KBOs and erode the Kuiper Belt beyond 50 AU. Kobayashi \& Ida (2001) elaborated on this idea and showed that perturbations from close stellar encounters (pericenter distance $\sim 200 \mathrm{AU}$ ) could have increased the $e$ 's and $i$ 's in the outer region of the planetesimal disk to the point where planetary accretion beyond a maximum semimajor axis $a_{\text {planet }}$ was inhibited. For a dense stellar cluster, $a_{\text {planet }}$ 

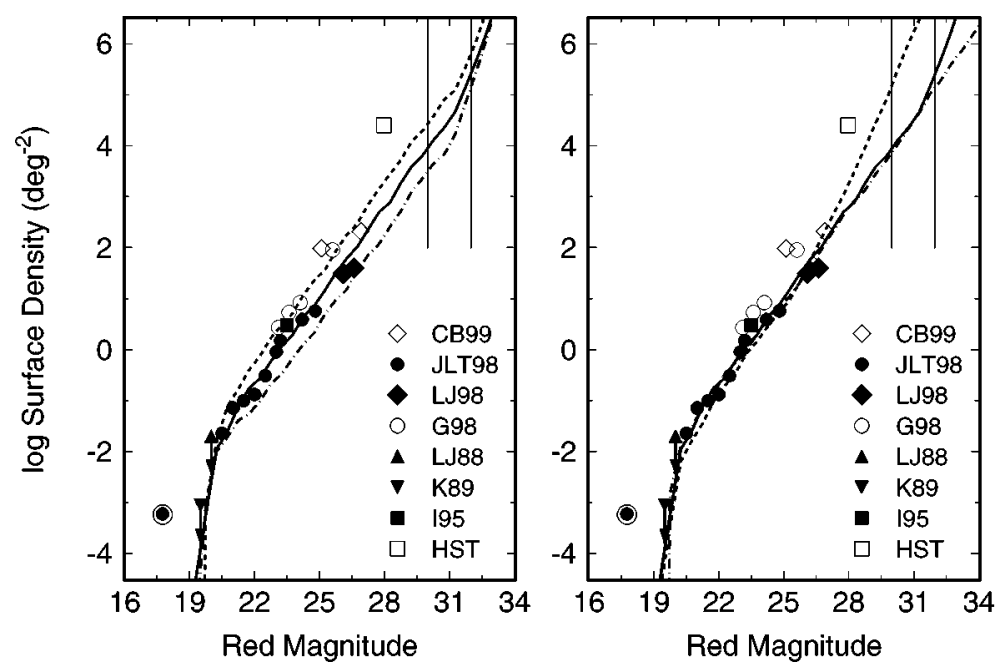

Figure 19 Comparison of model cumulative luminosity functions of KBOs with observations. The open circle with central dot indicates the position of Pluto for an adopted albedo of $4 \%$. The source of other observations are as follows: HST $=$ Cochran et al. (1998), I95 = Irwin et al. (1995), K89= Kowal (1989), LJ88 = Luu \& Jewitt (1988), G98 = Gladman et al. (1998), LJ98 = Luu \& Jewitt (1998), JLT98 = Jewitt et al. (1998), CB99 = Chiang \& Brown (1999). For clarity, error bars for each datum (typically a factor of 2-3) are not shown. $M_{M M S N}=7-17 M_{\oplus}=$ mass of the Minimum Mass Solar Nebula at $35 \mathrm{AU}$. (Left) Results from model with $e_{0}=10^{-3}$ and $M_{0}=$ $0.3 M_{M M S N}\left(\right.$ dot-dashed curve), $M_{0}=1 M_{M M S N}($ solid curve $), M_{0}=3 M_{M M S N}$ (dashed curve). (Right) Results from a model with $M_{0}=1 M_{M M S N}$ and $e_{0}=10^{-2}$ (dashed curve), $e_{0}=10^{-3}$ (solid curve), and $e_{0}=10^{-4}$ (dot-dashed curve). Figure adapted from Kenyon \& Luu (1999b).

was roughly the size of the solar system's planetary region ( 30-40 AU). Adams \& Laughlin (2001) investigated the effects on the Kuiper Belt if the solar system was formed in a stellar group large enough to contain a massive star, and found that all KBOs beyond $\sim 50$ AU would have attained large eccentricities from external perturbations ( $e>0.2$ for semimajor axis $a=50 \mathrm{AU}, e>0.4$ for $a=70 \mathrm{AU}$ ). As a result, $40 \%$ of the KBOs would have been removed by close encounters with Neptune. All these hypotheses, whether invoking planetary or stellar perturbers, share the outcome that the excited $e$ 's and $i$ 's of KBOs would have resulted in violent collisions and scattering events, adding to the collisional mass loss.

A rough history of the Kuiper Belt is thus one where the planetesimals originally had low relative velocities and nearly circular orbits (in order for growth to proceed). The velocity dispersion was of order $\Delta V=\left(i^{2}+e^{2}\right)^{1 / 2} V_{K}$, where $V_{K}$ is the local Keplerian orbital velocity. With $e=i[\mathrm{rad}]=0.001$, we find $\Delta V \approx$ 
$5 \mathrm{~m} \mathrm{~s}^{-1}$, similar to the escape velocity from a 5-km-radius planetesimal. These planetesimals grew by binary collisions, and as they grew, their velocity dispersion also increased due to mutual scattering. The increased velocity dispersion also meant stronger collisions and therefore a larger debris production rate. A significant fraction of this collision debris was in dust form, implying that the trans-Neptunian belt was originally very dusty, perhaps like the dust rings seen around some nearby main-sequence stars. Shortly after $10^{7} \mathrm{yrs}$, there were now bodies large enough (radius $\sim 300 \mathrm{~km}$ ) to excite the velocities of smaller planetesimals. At this point the dust content should rapidly increase, since collisions became disruptive instead of accumulative. The peak dust content should occur at $\sim 10^{8}$ years, right before nearby Neptune reached its final mass and began to erode the Kuiper Belt.

In short, it is clear that the Kuiper Belt has also undergone substantial dynamical and collisional erosion, and hypotheses abound that try to recreate its eventful past. Unexpectedly, the Kuiper Belt has turned out to be a test bed for theories of the Sun's birth environment.

\section{RELATIONSHIP WITH CIRCUMSTELLAR DISKS}

Since the discovery of the Kuiper Belt, it has often been postulated that dust disks around main sequence stars (age $\sim 100 \mathrm{Myr}$, e.g., $\beta$ Pic, Vega) are extrasolar analogs of the Kuiper Belt (e.g., Backman \& Paresce 1993). Dust rings of dimensions comparable to the Kuiper Belt have been resolved around several stars, such as HR4796A (Figure 21, see also Koerner et al. 1998) and $\epsilon$ Eridani (Greaves et al. 1998). Finally, dust has already been detected in our own Kuiper Belt by the spacecraft Voyager 1 and 2 (Gurnett et al. 1997). Both spacecraft detected dust impacts in the 30-50 AU region, and although Gurnett et al. did not identify the source of the impacts, Kuiper Belt dust seems to be the most plausible source. The dust production rate implied by the Voyager detections is $\sim 3 \times 10^{3} \mathrm{~kg} \mathrm{~s}^{-1}$ and the normal optical depth $\sim 10^{-7}$ (Jewitt \& Luu 1997).

There is thus strong evidence to link the Kuiper Belt to circumstellar disks, but since the evolution of circumstellar disks is still not well understood, it is not clear how the Kuiper Belt fits into this evolutionary scheme. The connection between the Kuiper Belt and circumstellar disks would be substantially elucidated if, for example, extrasolar KBOs were detected in circumstellar disks, or short of that, if the Kuiper Belt dust distribution could be compared with observations of circumstellar disks. According to studies of the orbital dynamics of Kuiper Belt dust (Liou et al. 1996, Liou \& Zook 1999), the latter might be feasible in the near future. Very small dust grains $(\leq 1 \mu \mathrm{m})$ originating in the Kuiper Belt are quickly blown out of the Solar System by radiation pressure, while the rest slowly spiral toward the Sun due to Poynting-Robertson and solar wind drag. During their journey they gravitationally interact with planets and can be scattered out of the 


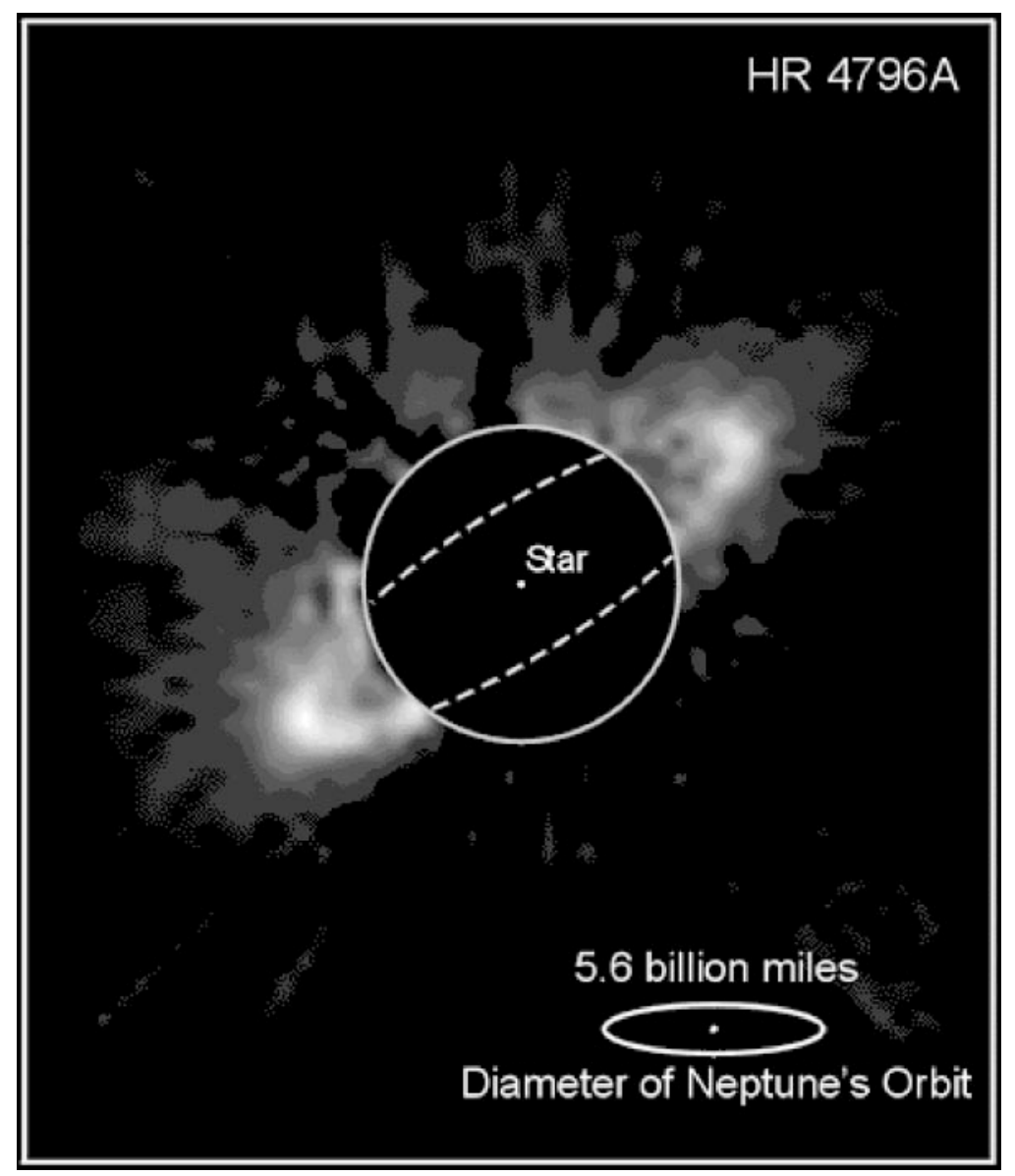

Figure 21 Near-infrared image of a dust ring around the star HR4796A, taken by NASA's Hubble Space Telescope. Figure courtesy of Brad Smith (University of Hawaii), Glenn Schneider (University of Arizona), and NASA.

solar system, trapped into mean motion resonances (Liou et al. 1996), or shattered by collisions with other Kuiper Belt grains or with interstellar dust (Yamamoto et al. 1998). Small $(<9 \mu \mathrm{m})$ dust grains generally reach the Sun and contribute to the zodiacal light. Intermediate-size (9-50 $\mu \mathrm{m}$ radius) grains are susceptible to collisional shattering by interstellar dust along the way, but much larger grains ( $>50 \mu \mathrm{m}$ radius) may survive these collisions and eventually also reach the Sun. Statistically, $80 \%$ of the grains are ejected from the Solar System, while $20 \%$ eventually reach the Sun and contribute to the zodiacal light.

Liou \& Zook (1999) pointed out that the distribution of circumstellar dust may provide another method by which to detect extrasolar planets (Figure 22). 
Dust tends to be trapped in mean motion resonances exterior to the outermost planet, and thus forms a giant circumsolar dust ring. The dust column density varies along the ring because different resonances have different resonance angles. The immediate vicinity of the planets should also be cleared of dust, due to ejection by the planets. It should be noted that the azimuthal dust variations may be washed out by interparticle collisions if the dust density is high. However, the optical depth of our Kuiper Belt dust is very low (Backman et al. 1995, Jewitt \& Luu 1997), so the brightness variations should still be preserved. In our solar system, the presence of the Kuiper Belt dust ring thus may be deduced by comparing the dust brightness at different longitudes (Figure 23). As seen from a space telescope, the minimum brightness would coincide with the direction where Neptune is located, and the maxima would appear close to the ecliptic, at $50-100^{\circ}$ ahead of or behind Neptune. A search for the Kuiper Belt dust signature (temperature $\sim 42 \mathrm{~K}$ ) in the COBE infrared sky map proved negative (Backman et al. 1995), but such longitudinal brightness variations may have been detected in the dust disk around the star $\epsilon$ Eri (Greaves et al. 1998, Liou et al. 2000).

Finally, Melnick et al. (2001) and Ford \& Neufeld (2001) pointed out that the vaporization of Kuiper Belt comets might explain the unexpected presence of detectable water vapor around late-type AGB stars. The ice mass $\left(\sim 10 M_{\oplus}\right)$ required to explain the water vapor abundance is much larger than the current mass of the Kuiper Belt, but is comparable to the inferred original mass (Kenyon \& Luu 1999a). Confirmation of this vaporization hypothesis would suggest that massive Kuiper Belts could survive for the entire main sequence lifetime of the central stars, even escaping the dramatic clearing phase that apparently removed $99 \%$ of the mass from our Kuiper Belt.

\section{FUTURE WORK}

Many external disks have large radial extents compared to the $50 \mathrm{AU}$ scale of the classical Kuiper Belt. In this regard, it is interesting to consider the possible structure of the outer Kuiper Belt, beyond the region that has so far been optically probed. One possibility, as already noted, is that the disk-like structure of the Kuiper Belt is simply absent beyond $50 \mathrm{AU}$, perhaps as a consequence of an ancient stellar encounter. Alternatively, it is also possible (e.g., Stern 1996) that the surface density of the Kuiper Belt drops at $50 \mathrm{AU}$ only to rise towards its primordial level at some larger radius. This speculation is not specific about the physical factors controlling the surface density, and offers no prediction of the distance at which a return to a larger density is expected. Naturally, it is difficult to rule it out. Observations show only that any wall in the surface density cannot be closer than $\sim 100$ AU. Because of the $R^{-4}$ dependence of the scattered sunlight, it will be difficult to study KBOs at 100's of AU and beyond. However, the planned Taiwan-America Occultation Survey (TAOS) experiment should provide 
an interesting constraint on the outer Belt and on the possible existence of a wall (Chen 1999). This experiment will photometrically monitor thousands of stars to search for occultations by intervening KBOs. The distance dependence of detectable occultations is modest (the size of the projected Fresnel diffraction spot, and hence of the smallest detectable KBO, varies only as $R^{1 / 2}$ ). Halley-sized (15 km in diameter) KBOs should be detectable many hundreds of AU from the sun, if they are present in sufficient abundance.

At the time of writing (August 2001), the Kuiper Belt has been amply probed in the magnitude range $20 \leq m_{R} \leq 25$, with sky coverage of a few hundred square degrees. Survey observations with the 8-m diameter SUBARU telescope on Mauna Kea have started, and should reach $\sim 28$ magnitude, probing Halley-nucleus sized objects in their natal region. At the other extreme, KBOs brighter than $m_{R} \sim 19$ are so rare (the expected surface density for $m_{R} \sim 19$ is $\sim 10^{-3} \mathrm{KBO}$ per square degree; see Figure 7) that large amounts of time on wide field telescopes are needed to find them.

A simple extrapolation from the current luminosity function to the entire area within $60^{\circ}$ of the ecliptic predicts that $\sim$ a few $\times 100 \mathrm{KBOs}$ brighter than $m_{R}=20$ will be found. Establishing the luminosity function at bright magnitudes provides clues to the accretion environment in the Kuiper Belt, especially in the late stages (Bailey 1994, Kenyon \& Luu 1999a), but, perhaps more importantly, the discovered bright KBOs provide the only opportunities to study the physical nature of KBOs as a group (see "Physical Properties" above). The fitted size distribution admits the possibility of finding more Pluto-sized (2000 km in diameter or larger) KBOs. If these objects (perhaps $\sim 5$ in number) still reside in the Solar System, they presumably inhabit the outer parts of the Kuiper Belt, because they would have been found otherwise.

The Large Synoptic Survey Telescope now being planned in the United States has the exploration of the Kuiper Belt as a major scientific priority (McKee \& Taylor 2001). This facility will survey the optical sky to magnitude 24 at least once every week. Through the use of sophisticated software, it will provide a continuing source of astrometric and photometric data on $\sim 10,000$ KBOs. These data will provide exquisite knowledge of the dynamical structure and contents of the trans-Neptunian solar system, rivalling that now available for the nearby main asteroid belt.

Ground-based observations have done much to reveal many of the gross properties of the Kuiper Belt, but such observations nevertheless suffer from limitations that result from being Earth-bound. Fortunately, these limitations can be rectified in the near future with space missions such as SIRTF and GAIA. SIRTF will allow the detection of the thermal radiation from $\sim 100$ KBOs. With simultaneous measurements at optical wavelengths, it will be possible to determine albedos and so to search for correlations between albedo, color and orbital properties (see Jewitt \& Luu 2001). Such correlations (or their absence) will tell us about the competing effects of collisions, cosmic ray bombardment, outgassing and compositional variations in the Kuiper Belt. GAIA is an all-sky astrometric and photometric survey 
that will create a precise three-dimensional map of the Milky Way and the Solar System. By dint of repeated astrometric and photometric observations of all objects brighter than $m_{V} \sim 20$, GAIA will yield the complete sample of all bright KBOs, free of all directional observational bias, and thus reveal the true three-dimensional distribution of the Kuiper Belt. GAIA's all-sky coverage will confirm the presence or absence of other Plutos and Charons in the Kuiper Belt, while its extremely precise astrometry of detected KBOs could reveal the presence of Kuiper Belt satellites from wobble about the barycenter. The sample of bright KBOs will be invaluable for detailed physical studies.

\section{ACKNOWLEDGMENT}

This work was supported by NASA's Origins of Solar Systems and Planetary Astronomy programs. We thank R. Hoogerwerf for help with the figures, and J. Kleyna for working during his vacation.

\section{The Annual Review of Astronomy and Astrophysics is online at http://astro.annualreviews.org}

\section{LITERATURE CITED}

Adams FC, Laughlin G. 2001. Icarus 150:15162

Allen RL, Bernstein GM, Malhotra R. 2001. Astrophys. J. 549:L241-44

Backman DE, Dasgupta A, Stencel RE. 1995. Astrophys. J. 450:L35-38

Backman DE, Paresce F. 1993. Main-sequence stars with circumstellar solid material-The VEGA phenomenon. In Protostars and Planets III, ed. E Levy, J Lunine, pp. 1253-304. Tucson: Univ. Ariz. Press

Bailey ME. 1994. Formation of the outer solar system bodies: Comets and planetesimals. In Asteroids, Comets, Meteors 1993. Proc. IAU Symp. 160, ed. A Milani, M Di Martino, A Cellino, pp. 443-59. Dordrecht: Kluwer

Barucci MA, Romon J, Doressoundiram A, Tholen DJ. 2000. Astron. J. 120:496-500

Bockelee-Morvan D, Lellouch E, Biver N, Paubert G, Bauer J, et al. 2001. Astron. Astrophys. 377:343-53

Brown ME. 2001. Astron. J. 121:2804-14

Brown ME, Blake GA, Kessler JE. 2000. Astrophys. J. 543:L163-65
Brown RH, Cruikshank DP, Pendleton Y. 1999. Astrophys. J. 519:L101-4

Brown ME, Kulkarni SR, Liggett TJ. 1997. Astrophys. J. 490:L119-22

Chen WP. 1999. Census of Kuiper Cometsthe TAOS Project. In Observational Astrophysics in Asia and its Future. Proc. East Asian Meetg. Astron., 4th, Kunming, ed. PS Chen, pp. 181. Yunnan Observatory. Beijing: Chinese Acad. Sci.

Chiang EI, Brown ME. 1999. Astron. J. 118: 1411-22

Clark RN. 1981a. J. Geo. Res. 86:3087-96

Clark RN. 1981b. J. Geo. Res. 86:3074-86

Cochran AL, Levison HF, Stern SA, Duncan MJ. 1995. Astrophys. J. 455:342-46

Cochran AL, Levison HF, Tamblyn P, Stern SA, Duncan MJ. 1998. Astrophys. J. 503:L8993

Collander-Brown SJ, Fitzsimmons A, Fletcher E, Irwin MJ, Williams IP. 1999. MNRAS 308:588-92

Crovisier J, Brooke TY, Leech K, BockeleeMorvan D, Lellouch E, et al. 2000. The 
thermal infrared spectra of comets HaleBopp and 103P/Hartley 2 observed with the Infrared Space Observatory. In Thermal Emission Spectroscopy and Analysis of Dust, Disks, and Regoliths, Astron. Soc. Pac. Conf. Ser., ed. M Sitko, A Sprague, D Lynch, 196:109-17

Cruikshank DP, Roush TL, Bartholomew MJ, Moroz LV, Geballe TR, et al. 1998. Icarus 135:389-407

Cuillandre J-C, Luppino G, Starr B, Isani S. 2000. Performance of the CFH12K: a $12 \mathrm{~K}$ by $8 \mathrm{~K} \mathrm{CCD} \mathrm{mosaic} \mathrm{camera} \mathrm{for} \mathrm{the} \mathrm{CFHT} \mathrm{prime}$ focus. In Optical and IR Telescope Instrumentation and Detectors, Proc. SPIE, ed. M Iye, AF Moorwood, 4008:1010-21. Bellingham, WA: SPIE

Davies JK. 2001. Beyond Pluto: Exploring the Outer Limits of the Solar System. Cambridge: Cambridge Univ. Press

Davies JK, Green S, McBride N, Muzzerall E, Tholen DJ, et al. 2000. Icarus 146:253-62

De Sanctis MC, Capria MT, Coradini A. 2001. Astron. J. 121:2792-99

Dones L. 1997. Origin and evolution of the Kuiper Belt. In From Stardust to Planetesimals, Astron. Soc. Pac. Conf. Ser., Vol. 122, ed. YJ Pendleton, AGGM Tielens, ML Savage, pp. 347-65. San Francisco: Astron. Soc. Pac.

Dones L, Gladman B, Melosh HJ, Tonks WB, Levison HF, Duncan M. 1999. Icarus 142:509-24

Dohnanyi JS. 1969. J. Geophys. Res. 74:253154

Duncan M, Quinn T, Tremaine S. 1988. Astrophys. J. 328:69-73

Duncan MJ, Levison HF. 1997. Science 276:1670-72

Duncan MJ, Levison HF, Budd SM. 1995. Astron. J. 110:3073-81

Edgeworth KE. 1943. J. Br. Astron. Soc. 53:181-88

Edgeworth KE. 1949. MNRAS 109:600-9

Eggers S, Keller HU, Kroupa P, Markiewicz WJ. 1997. Planet. Space Sci. 45:1099-104

Elliot JL, Kern SD, Osip DJ, Burles S. 2001. IAU circular 1733
Farinella P, Davis D. 1996. Science 273:938-41

Farinella P, Davis DR, Stern SA. 2000. Formation and Collisional Evolution of the Edgeworth-Kuiper Belt. See Mannings et al. 2000, pp. 1255-82

Farinella P, Milani A, Nobili AM, Valsacchi GB. 1979. Moon Planets 20:415-21

Fernandez JA. 1980. MNRAS 192:481-91

Fernandez JA, Brunini A. 2000. Icarus 145: 580-90

Fernandez JA, Ip W-H. 1984. Icarus 58:109-20

Fernandez YR, Jewitt DC, Sheppard SS. 2001. Astrophys. J. 553:197-200

Ferrin I, Rabinowitz D, Schaefer B, Snyder J, Ellman N, et al. 2001. Astrophys. J. 548: L243-47

Fitzsimmons A, Jewitt DC, West R. 2000. Minor Bodies in the Outer Solar System. Berlin: Springer-Verlag

Ford K, Neufeld D. 2001. Astrophys. J. 557: 113-16

Friedland L. 2001. Astrophys. J. 547:L75-79

Gladman B, Kavelaars JJ, Nicholson PD, Loredo TJ, Burns JA. 1998. Astron. J. 116: 2042-54

Gladman B, Kavelaars JJ, Petit J-M, Morbidelli A, Holman MJ, Loredo T. 2001. Astron. J. 122:1051-66

Goldreich P, Murray N, Longaretti PY, Banfield D. 1989. Science 245:500-4

Greaves JS, Holland WS, Moriarty-Schieven G, Jenness T, Dent WRF, et al. 1998. Astrophys. J. 506:L133-37

Green SF, McBride N, O'Ceallaigh DP, Fitzsimmons A, Williams IP, Irwin MJ. 1997. MNRAS 290:186-92

Gurnett DA, Ansher JA, Kurth WS, Granroth LJ. 1997. Geophys. Res. Lett. 24:3125-28

Hahn G, Bailey ME. 1990. Nature 348:132-36

Hahn JM, Malhotra R. 1999. Astron. J. 117: 3041-53

Hainaut OR, Delahodde CE, Boehnhardt H, Dotto E, Barucci MA, et al. 2000. Astron. Astrophys. 356:1076-88

Hamid SE, Marsden BG, Whipple FL. 1968. Astron. J. 73:727-28

Harrington RS, van Flandern TC. 1979. Icarus 39:131-36 
Hartmann W, Tholen DJ, Meech K, Cruikshank DP. 1990. Icarus 83:1-15

Hayashi C, Nakazawa K, Nakagawa Y. 1985. Formation of the solar system. In Protostars and Planets II, ed. DC Black, MS Matthews, pp. 1100-53. Tucson: Univ. Ariz. Press

Hogg DW, Quinlan GD, Tremaine S. 1991. Astron. J. 101:2274-86

Holman M, Wisdom J. 1993. Astron. J. 105:1987-99

Hunt GR. 1977. Geophysics 42:501-13

Ida S, Bryden G, Lin DNC, Tanaka H. 2000a. Astrophys. J. 534:428-45

Ida S, Larwood J, Burkert A. 2000b. Astrophys. J. 528:351-56

Ip W-H. 1989. Icarus 80:167-78

Ip W-H, Fernandez JA. 1991. Icarus 92:185-93

Ip W-H, Fernandez JA. 1997. Astron. Astrophys. 324:778-84

Irwin M, Tremaine S, Zytkow AN. 1995. Astron. J. 110:3082-92

Jewitt DC. 1990. In Comets in the Post-Halley Era, ed. R Newburn, M Neugebauer, J Rahe, pp. 19-65. Dordrecht: Kluwer

Jewitt DC. 1999. Annu. Rev. Earth Planet. Sci. 27:287-312

Jewitt DC. 2002. Astron J. In press

Jewitt DC, Aussel H, Evans A. 2001. Nature 411:446-47

Jewitt DC, Luu JX. 1993. Nature 362:73032

Jewitt DC, Luu JX. 1997. The Kuiper Belt. In From Stardust to Planetesimals, ed. Y Pendleton, AGG Tielens, pp. 335-46. San Francisco: Astron. Soc. Pac.

Jewitt DC, Luu JX. 2000. Physical Nature of the Kuiper Belt. See Mannings et al. 2000, pp. 1201-29

Jewitt DC, Luu JX. 2001. Astron. J. 122:2099_ 114

Jewitt DC, Luu JX, Chen J. 1996. Astron. J. 112:1225-38

Jewitt DC, Luu JX, Trujillo C. 1998. Astron. J. 115:2125-35

Jewitt DC, Matthews H. 1999. Astron. J. 117:1056-62

Jewitt DC, Sheppard SS. 2002. Astron. J. In press
Kavelaars J, Petit J, Gladman B, Holman M. 2001. IAU Circ. 7749

Kenyon SJ, Luu JX. 1998. Astron. J. 115:213660

Kenyon SJ, Luu JX. 1999a. Astron. J. 118: 1101-19

Kenyon SJ, Luu JX. 1999b. Astrophys. J. 526: 465-70

Kern SD, McCarthy DW, Buie MW, Brown RH, Campins H, Rieke M. 2000. Astrophys. J. 542:L155-59

Kobayashi H, Ida S. 2001. The effects of a stellar encounter on a planetesimal disk. In Am. Astron. Soc., 33rd Div. of Planet Sci. Meet. 15.06 (Abstr.)

Koerner DW, Ressler ME, Werner MW, Backman DE. 1998. Astrophys. J. 503:L83-87

Kossacki KJ, Leliwa-Kopystynski J. 1993. Planet. Space Sci. 41:729-41

Kowal C. 1989. Icarus 77:118-23

Kroupa P. 1995. MNRAS 277:1507-21

Kroupa P. 1998. MNRAS 298:231-42

Kuiper GP. 1951. On the origin of the solar system. In Astrophysics, ed. JA Hynek, pp. 357424. New York: McGraw-Hill

Lamy PL, Toth I, Weaver HA, Delahodde C, Jorda L, A'Hearn MF. 2000. The nucleus of 13 short-period comets. In Am. Astron. Soc., 32nd Div. Planet. Sci. Meet. 36.04 (Abstr.)

Larsen JA, Gleason AE, Danzl NM, Descour AS, McMillan RS, et al. 2001. Astron. J. 121:562-79

Leonard FC. 1930. The New Planet Pluto. Leaflet Astron. Soc. Pacific No. 30, Aug., pp. 121-24

Levison HF, Duncan MJ. 1990. Astron. J. 100:1669-75

Levison HF, Duncan MJ. 1993. Astrophys. J. 406:L35-38

Levison HF, Duncan MJ. 1994. Icarus 108:1836

Licandro J, Oliva E, Di Martino M. 2001. Astron. Astrophys. 373:L29-32

Liou J-C, Zook HA. 1999. Astron. J. 118:58090

Liou J-C, Zook HA, Dermott SF. 1996. Icarus 124:429-40 
Liou J-C, Zook HA, Greaves JS, Holland WS. 2000. Does a planet exist in $\epsilon$ Eridani? A comparison between observations numerical simulations. In 31st Ann. Lunar Planet. Sci. Conf. 1416 (Abstr.)

Lissauer JJ, Pollack JB, Wetherill GW, Stevenson DJ. 1996. In Neptune and Triton, ed. DP Cruikshank, MS Matthews, AM Schumann, pp. 37-108. Tucson: Univ. Ariz. Press

Lisse C, A'Hearn M, Hauser M, Kelsall T, Lien D, et al. 1998. Astrophys. J. 496:971-91

Luu JX, Jewitt DC. 1988. Astron. J. 95:1256-62

Luu JX, Jewitt DC. 1990. Astron. J. 100:91332

Luu JX, Jewitt DC. 1996. Astron. J. 112:2310 18

Luu JX, Jewitt DC. 1998. Astrophys. J. 502:9194

Luu JX, Jewitt DC, Trujillo CA. 2000. Astrophys. J. 531:L151-54

Luu JX, Marsden B, Jewitt DC, Trujillo CA, Hergenrother C, et al. 1997. Nature 387:57375

Lyttleton RA. 1936. MNRAS 97:108-15

MacFarland J. 1996. Vistas Astron. 40:34354

Malhotra R. 1993. Nature 365:819

Malhotra R. 1995. Astron. J. 110:420-29

Malhotra R, Duncan MJ, Levison HJ. 2000. Dynamics of the Kuiper Belt. See Mannings et al. 2000, pp. 1231-54

Mannings V, Boss AP, Russell SS, eds. 2000. Protostars and Planets IV. Tucson: Univ. Ariz. Press

Marcialis RL, Buratti BJ. 1993. Icarus 104: 234-43

McCaughrean M, O’Dell R. 1996. Astron. J. 111:1977-86

McKee C, Taylor J. 2001. Astronomy \& Astrophysics in the New Millennium. Washington, DC: Natl. Acad. Press

McKinnon WB. 1989. Astrophys. J. 344:L4143

McKinnon W, Chapman C, Housen K. 1991. In Uranus, ed. J Bergstrahl, E Miner, M Matthews, pp. 620-92. Tucson: Univ. Arizona Press
McKinnon WB, Leith AC. 1995. Icarus 118: 392-413

Meech K, Belton MJS. 1990. Astron. J. 100: 1323-38

Melnick GJ, Neufeld DA, Ford KES, Hollenbach DJ, Ashby MLN. 2001. Nature 412:160-63

Merline WJ, Close LM, Dumas C, Shelton JC, Menard F, et al. 2000. Discovery of Companions to Asteroids 762 Pulcova and 90 Antiope by Direct Imaging. In Am. Astron. Soc. 32nd Div. Planet. Sci. Mtg. 13.06 (Abstr.)

Morbidelli A, Thomas F, Moons M. 1995. Icarus 118:322-40

Morbidelli A, Valsecchi GB. 1997. Icarus 128:464-68

Moroz LV, Arnold G, Korochantsev AV, Wésch R. 1998. Icarus 134:253-68

Nagasawa M, Ida S. 2000. Astron. J. 120:331122

Oort JH. 1950. Bull. Astron. Inst. Netherlands 11:91-110

Petit J-M, Morbidelli A, Valsecchi GB. 1999. Icarus 141:367-87

Prialnik D. 2000. See Fitzsimmons et al. 2000. pp. 33-50

Prialnik D, Podolak M. 1995. Icarus 117:42030

Rickman H, Kamel L, Festou MC, Froeschle C. 1987. Estimates of masses, volumes and densities of short-period comet nuclei. In Proc. Int. Symp. Diversity Similarity Comets, pp. 471-81. Noordwijk: Eur. Space Agency

Romanishin W, Tegler SC. 1999. Nature 398: 129-32

Safronov VS. 1969. Evolution of the Protoplanetary Cloud and Formation of the Earth and Planets. Moscow: Nauka. Transl. Israel Prog. Sci. Transl., 1972. NASA TT F677

Shul'man LM. 1972. The chemical composition of cometary nuclei. In The Motion, Evolution of Orbits, and Origin of Comets. Proc. IAU Symp. 145, ed. G Chebotarev, E Kazimirchak-Polonskaia, B Marsden, pp. 265-70. Dordrecht: Reidel

Schulz R. 2001. Astron. Astrophys. Rev. In press Sheppard SS, Jewitt DC, Trujillo CA, Brown 
MJI, Ashley MCBA. 2000. Astron. J. 120: 2687-94

Stacey FD. 1969. Physics of the Earth, pp. 23962. New York: Wiley

Stern SA. 1996. Astron. J. 112:1203-11

Stern SA, Colwell JE. 1997. Astron. J. 114:84149

Strazzulla G, Johnson RE. 1991. In Comets in the Post-Halley Era, ed. R Newburn Jr, M Neugebauer, J Rahe, pp. 243-76. Astrophys. Space Sci. Lib. Vol. 167. Dordrecht: Kluwer Acad.

Tegler SC, Romanishin W. 1998. Nature 392: 49-50

Tegler SC, Romanishin W. 2000. Nature 407: 979-81

Tholen D, Buie M. 1997. Bulk properties of Pluto and Charon. In Pluto and Charon, ed. SA Stern, DJ Tholen, pp. 193-220. Tucson: Univ. Ariz. Press

Tombaugh C. 1961. The trans-Neptunian planet search. In Planets and Satellites, ed. G Kuiper, B Middlehurst, pp. 12-30. Chicago: Univ. Chicago Press

Torbett M. 1989. Astron. J. 98:1477-81

Toth I. 1999. Icarus 141:420-25
Trujillo CA, Brown ME. 2001. Astrophys. J. 554:L95-98

Trujillo CA, Brown ME. 2002a. Astrophys. J. Lett. In press

Trujillo CA, Brown ME. 2002b. IAC Circ. 7787

Trujillo CA, Jewitt DC. 1998. Astron. J. 115: 1680-87

Trujillo CA, Jewitt DC, Luu JX. 2000. Astrophys. J. 529:L103-6

Trujillo CA, Jewitt DC, Luu JX. 2001a. Astron. J. 122:457-73

Trujillo CA, Luu JX, Bosh AS, Elliot JL. 2001b. Astron. J. Astron. J. 122:2740-48

Veillet C, Doressoundiram A, Shapiro J, Morbidelli A. 2001. IAU Circ. 7610

Wetherill GW. 1980. Annu. Rev. Astron. Astrophys. 18:77-113

Wetherill GW, Stewart GR. 1993. Icarus 106: 190-209

Wilson PD, Sagan C, Thompson WR. 1994. Icarus 107:288-303

Woolfson MM. 1999. MNRAS 304:195-98

Yamamoto S, Mukai T. 1998. Astron. Astrophys. 329:785-91

Yu Q, Tremaine S. 1999. Astron. J. 118:187381 


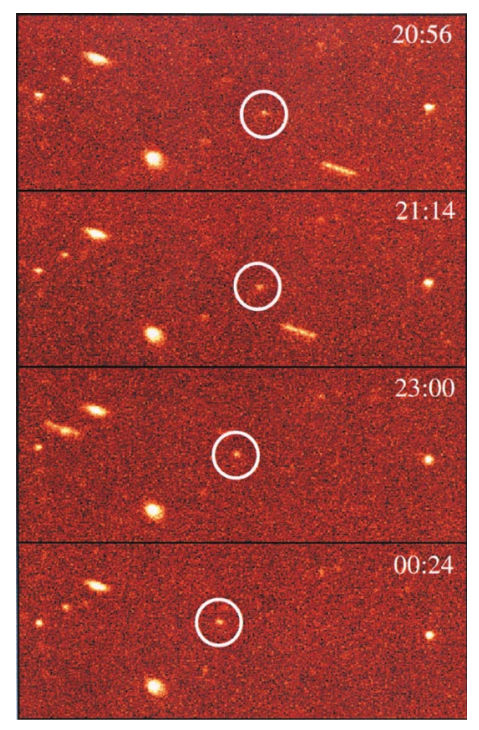

Figure 1 Discovery images of the first Kuiper Belt object 1992 QB $_{1}$, taken on 1992 August 30, at the University of Hawaii $2.2 \mathrm{~m}$ telescope. The four CCD images (UT time indicated in the upper right hand corner) show $\mathrm{QB}_{1}$ (circled) slowly moving left against the background stars and galaxies. The elongated trail in the top 3 images is an asteroid (note the slow motion of $\mathrm{QB}_{1}$ compared to the motion of the asteroid). Figure from Jewitt \& Luu (1993).

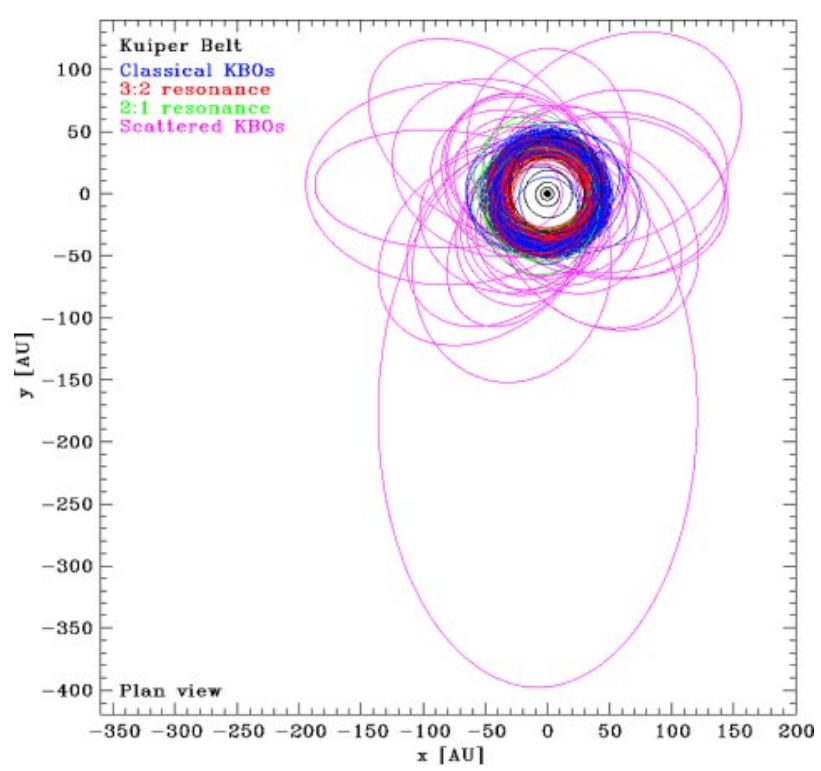

Figure 2 Plan view of the Kuiper Belt (as seen from the North ecliptic pole) showing the orbits of KBOs as of June 2001. The orbits are color-coded according to the legend: blue $=$ Classical KBOs, red $=3: 2$ resonance, green $=2: 1$ resonance, pink $=$ Scattered KBOs. Innermost circle is Jupiter's orbit. The region shown is $560 \times 560$ AU. 


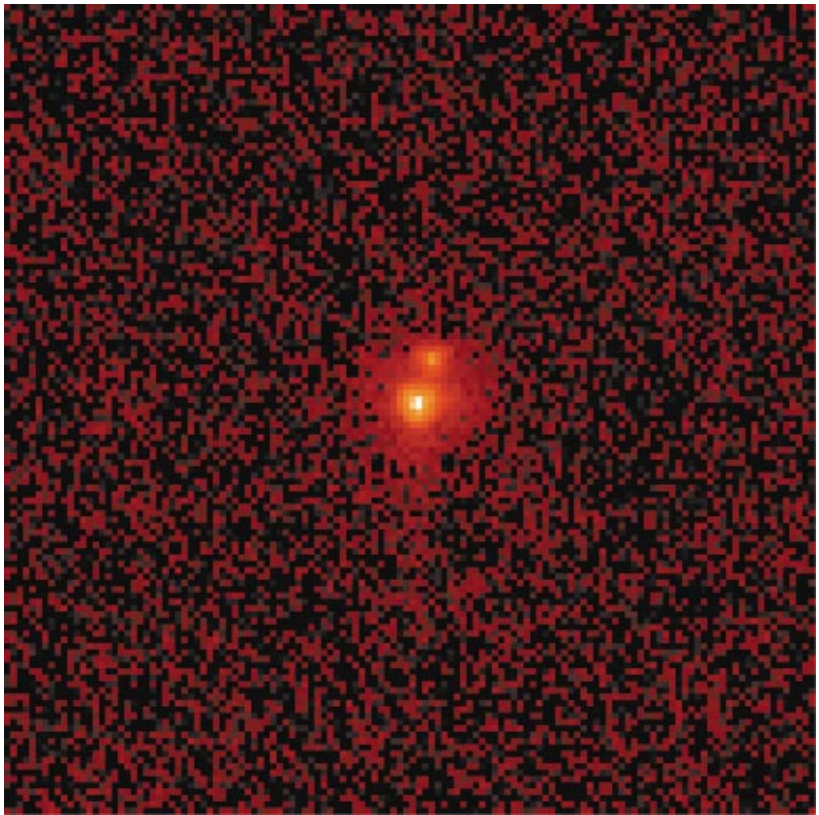

Figure 15 CCD image of the binary KBO $1999 \mathrm{TC}_{36}$ taken with the Hubble Space Telescope, showing the binary and secondary clearly separated by $\sim 0.37$ arcsec. Image courtesy of C. Trujillo (Caltech).

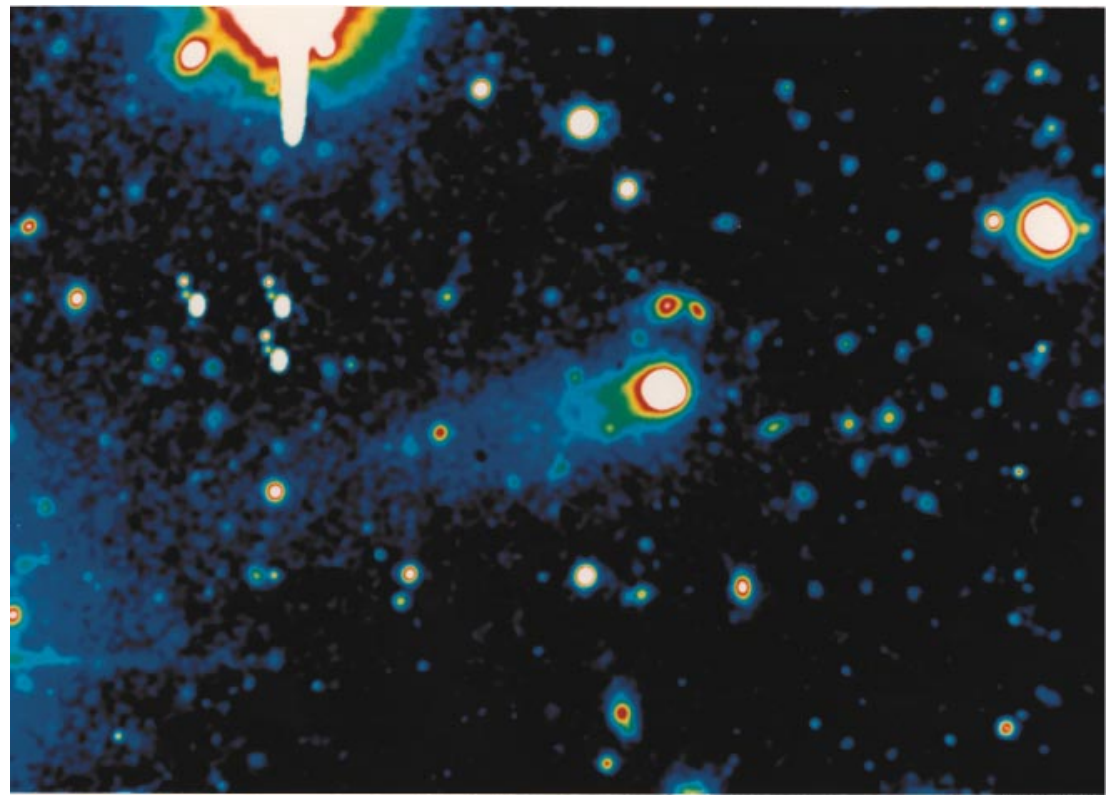

Figure 16 Centaur 2060 Chiron, with cometary tail (pointing toward lower left hand corner). Image is $\sim 2$ arcmin wide. 

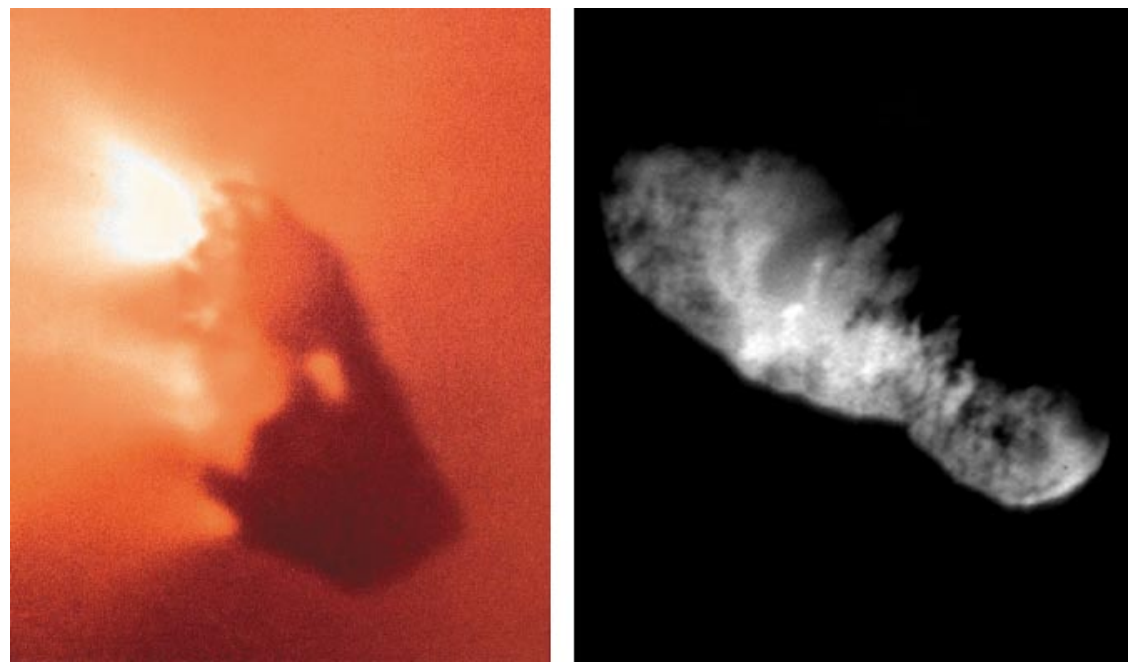

Figure 17 (a) The $16 \times 8 \times 8 \mathrm{~km}$ nucleus of comet Halley. Photo taken by the Halley Multicolour Camera onboard ESA's Giotto spacecraft (copyright Max-Planck-Institut fuer Aeronomie, Lindau/Harz, Germany). Figure courtesy of Dr. H. U. Keller. (b) The $8 \mathrm{~km}$-long nucleus of comet Borrelly. Photo taken by the Deep Space 1 spacecraft in September 2001. Figure courtesy of NASA/JPL.
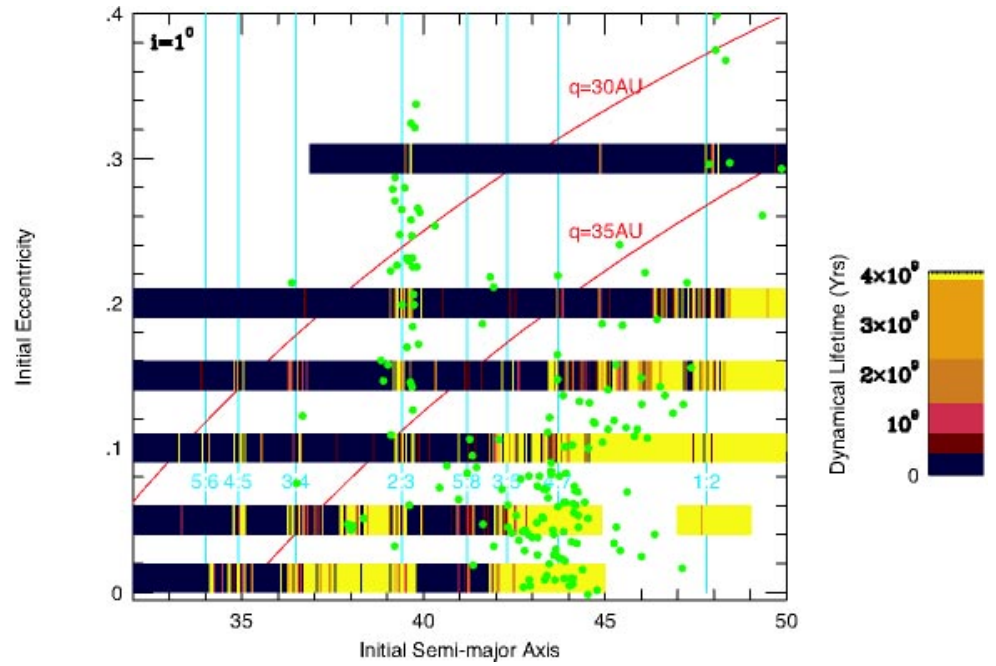

Figure 20 Dynamical lifetimes in the Kuiper Belt. Each narrow vertical strip at a given semimajor axis $a$ and eccentricity $e$ represents the lifetime of a test particle with the given initial $a$ and $e$ (initial inclination $i=1^{\circ}$ ). The strips are color-coded according to the lifetimes. Yellow denotes objects that survive for $4 \mathrm{Gyr}$, and progressively darker regions denote progressively shorter dynamical lifetimes. The green circles represent known KBOs observed at more than 1 opposition (as of 2001 Aug 1). The long blue vertical lines mark the locations of the important Neptune mean motion resonances. The red curves denote constant perihelion distance $q$. Figure adapted from Duncan et al. (1995). 


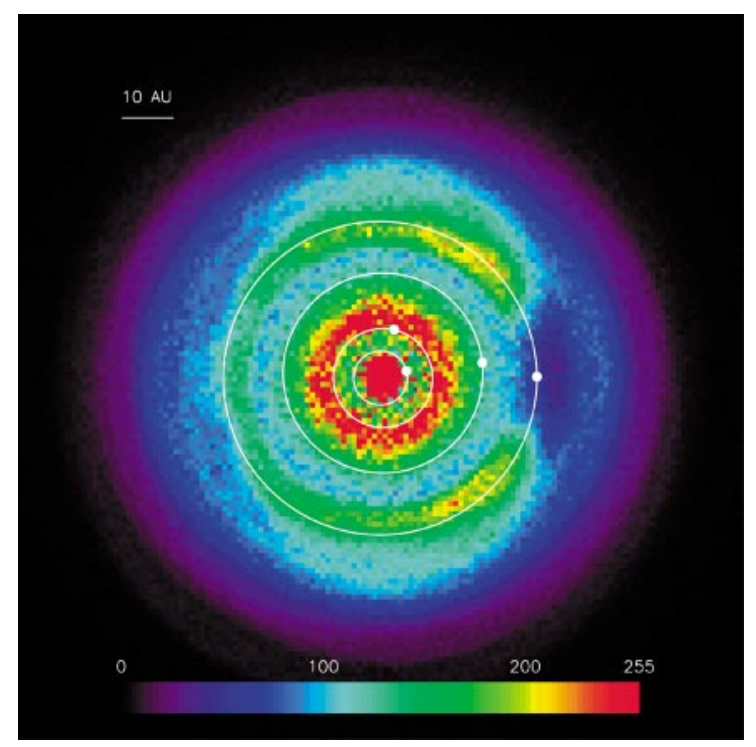

Figure 22 The calculated brightness of $23 \mu \mathrm{m}$ dust in the solar system (face-on view) in false colors. The orbits and positions of the four giant planets are shown in yellow. Colors correspond to the number of dust particles per $\mathrm{AU}^{2}$ (red is brightest, purple darkest). Figure courtesy of Liou \& Zook (1999).

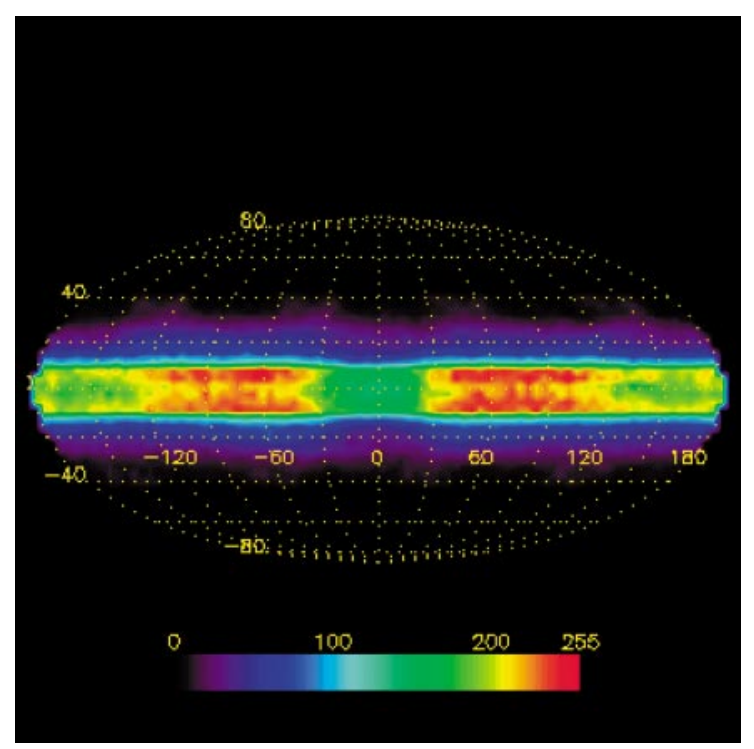

Figure 23 Brightness variations of $23 \mu \mathrm{m}$ Kuiper Belt dust grains outside $20 \mathrm{AU}$, as viewed from the Sun. The vertical axis is the ecliptic latitude, and the horizontal axis the longitude with respect to Neptune (longitude $0^{\circ}$ ). Notice the two enhancements at $50^{\circ}-100^{\circ}$ ahead and behind Neptune. Figure courtesy of Liou \& Zook (1999). 
胥 Annual Review of Astronomy and Astrophysics

Volume 40, 2002

\section{CONTENTS}

FRONTISPIECE, Edwin E. Salpeter

A Generalist LoOKS BaCK, Edwin E. Salpeter

xii

UltRA-COMPACT HII REGIONS AND MASSIVE STAR Formation, Ed Churchwell

KUIPER BELT OBJECTS: RELICS FROM THE ACCRETION DISK OF THE Sun, Jane X. Luu and David C. Jewitt

TheOry of Giant Planets, W. B. Hubbard, A. Burrows, and J. I. Lunine

Theories of Gamma-Ray Bursts, P. Mészáros

COSMIC MiCROWAVE BACKGROUND ANISOTROPIES, Wayne $\mathrm{Hu}$ and Scott Dodelson

Stellar RAdio Astronomy: PROBing Stellar ATMOSPHERES FROM PROTOSTARS TO GIANTS, Manuel Güdel

Modified NeWtonian Dynamics as an Alternative to DARK MATTER, Robert H. Sanders and Stacy S. McGaugh

Cluster Magnetic Fields, C. L. Carilli and G. B. Taylor

The Origin of Binary Stars, Joel E. Tohline

RADIO EMISSION FROM SUPERNOVAE AND GAMMA-RAY Bursters, Kurt W. Weiler, Nino Panagia, Marcos J. Montes, and Richard A. Sramek

Shapes ANd Shaping of Planetary NebUlae, Bruce Balick and Adam Frank

The New GalaXy: Signatures of its Formation, Ken Freeman and Joss Bland-Hawthorn

The Evolution of X-Ray Clusters of GalaXies, Piero Rosati, Stefano Borgani, and Colin Norman

LyMAN-BREAK GalaXIES, Mauro Giavalisco

COSMOLOGY WITH THE SUNYAEV-ZEL'DOVICH EFFECT, John E. Carlstrom, Gilbert P. Holder, and Erik D. Reese 


\section{INDEXES}

Subject Index

Cumulative Index of Contributing Authors, Volumes 29-40

Cumulative Index of Chapter Titles, Volumes 29-40

\section{ERRATA}

An online log of corrections to Annual Review of Astronomy and Astrophysics chapters (if any, 1997 to the present)

may be found at http://astro.annualreviews.org/errata.shtml 